\title{
The Feigin Tetrahedron ${ }^{\star}$
}

Dylan RUPEL

Department of Mathematics, Northeastern University, Boston, MA 02115, USA

E-mail:d.rupel@neu.edu

URL: http://www.northeastern.edu/drupel/

Received September 11, 2014, in final form March 03, 2015; Published online March 19, 2015

http://dx.doi.org/10.3842/SIGMA.2015.024

\begin{abstract}
The first goal of this note is to extend the well-known Feigin homomorphisms taking quantum groups to quantum polynomial algebras. More precisely, we define generalized Feigin homomorphisms from a quantum shuffle algebra to quantum polynomial algebras which extend the classical Feigin homomorphisms along the embedding of the quantum group into said quantum shuffle algebra. In a recent work of Berenstein and the author, analogous extensions of Feigin homomorphisms from the dual Hall-Ringel algebra of a valued quiver to quantum polynomial algebras were defined. To relate these constructions, we establish a homomorphism, dubbed the quantum shuffle character, from the dual Hall-Ringel algebra to the quantum shuffle algebra which relates the generalized Feigin homomorphisms. These constructions can be compactly described by a commuting tetrahedron of maps beginning with the quantum group and terminating in a quantum polynomial algebra. The second goal in this project is to better understand the dual canonical basis conjecture for skewsymmetrizable quantum cluster algebras. In the symmetrizable types it is known that dual canonical basis elements need not have positive multiplicative structure constants, while this is still suspected to hold for skew-symmetrizable quantum cluster algebras. We propose an alternate conjecture for the symmetrizable types: the cluster monomials should correspond to irreducible characters of a KLR algebra. Indeed, the main conjecture of this note would establish this "KLR conjecture" for acyclic skew-symmetrizable quantum cluster algebras: that is, we conjecture that the images of rigid representations under the quantum shuffle character give irreducible characters for KLR algebras. We sketch a proof in the symmetric case giving an alternative to the proof of Kimura-Qin that all non-initial cluster variables in an acyclic skew-symmetric quantum cluster algebra are contained in the dual canonical basis. With these results in mind we interpret the cluster mutations directly in terms of the representation theory of the KLR algebra.
\end{abstract}

Key words: cluster algebra; Hall algebra; quantum group; quiver Hecke algebra; KLR algebra; dual canonical basis; Feigin homomorphism; categorification

2010 Mathematics Subject Classification: 13F60; 16G20; 17B37; 20G42

\section{Introduction}

In the struggle to understand the quantum groups and their duals, the quantized enveloping algebras, one searches for concrete realizations of these algebras, either by generators and relations or via embeddings, or more generally simply homomorphisms, into "nicer" algebras. This note grew out of an attempt to understand three well-known and well-studied examples, namely the embedding into a (dual) Hall-Ringel algebra, the embedding into a quantum shuffle algebra, and the Feigin homomorphisms to quantum polynomial algebras.

The embeddings of the (positive part of) quantized Kac-Moody algebras into Hall-Ringel algebras originated with the incredible insights of Ringel, for the finite-types in [29] and fully

\footnotetext{
${ }^{\star}$ This paper is a contribution to the Special Issue on New Directions in Lie Theory. The full collection is available at http://www.emis.de/journals/SIGMA/LieTheory2014.html
} 
realized in [30]. This is merely the tip of a fantastic geometric iceberg discovered by Lusztig [25] that has lead to a deep understanding of certain "canonical" bases in the quantized Kac-Moody algebras.

A careful study of multiplicative properties of these bases helped motivate Fomin and Zelevinsky in the definition of cluster algebras. In the hopes of gaining a combinatorial grasp of the structure of the canonical basis they provided the definition of a recursively, combinatorially defined algebra with a deep conjecture in mind: certain "cluster monomials" appearing in this construction should be identifiable with (dual) canonical basis vectors. This motivating conjecture has been established so far in a very restricted set of cases by Lampe [20, 21] and Kimura-Qin [16]. One aim of this note is to shed some additional light on this "dual canonical basis conjecture".

The quantized Kac-Moody algebras are most compactly described by generators and relations or better as a certain quotient of a free algebra with an unconventional (dare I say twisted) multiplication defined on its tensor square. Dualizing this construction leads to an embedding of the quantum group into the quantum shuffle algebra and thus, via an isomorphism, an embedding of the quantized Kac-Moody algebra. This is the tip of yet another (not entirely unrelated) iceberg, this one categorical [14, 15, 32, 33], which provides another description of these positively amazing bases of the quantum group and quantized Kac-Moody algebra.

Our third major tool for studying quantized Kac-Moody algebras are certain algebra homomorphisms to quantum polynomial rings suggested by B. Feigin during a talk at RIMS in 1992 (thus we refer to these as Feigin homomorphisms). There are actually infinitely many Feigin homomorphisms acting on a given quantized Kac-Moody algebra, one corresponding to each finite sequence of simple roots. For finite-types, we get an embedding whenever the sequence corresponds to a reduced word for the longest element of the corresponding Weyl group. In general a Feigin homomorphism has a very large kernel, a feature which we hope will shed some light on the relationship of KLR characters to quantum cluster algebras. The Feigin homomorphisms, as Feigin predicted, turn out to be the essential tool $[2,10,12]$ for studying the skew-field of fractions of the quantized Kac-Moody algebras.

The relationship between the first and third approach was studied by Berenstein and the author in [3] where Feigin homomorphisms were extended to the dual Hall-Ringel algebra for representations of an acyclic valued quiver. Applications to quantum cluster algebras were a central result in [3] where the images of rigid objects are identified with quantum cluster characters describing non-initial cluster variables of a corresponding acyclic quantum cluster algebra $[28,34,35]$. This also provided the essential tool for understanding a certain "twist automorphism" of the quantum cluster algebra, it would be interesting to use the constructions/conjectures of this note to interpret the twist in terms of the representation theory of KLR algebras.

The main result of the current work is to complete the above tetrahedron. We begin in Section 6 by extending Feigin homomorphisms to the quantum shuffle algebra. Section 7 introduces a homomorphism, the quantum shuffle character, from the dual Hall-Ringel algebra to the quantum shuffle algebra making all of the relevant triangles commute.

A secondary goal of this project is to understand the dual canonical basis conjecture for quantum cluster algebras. The generalized Feign homomorphism of [3] produces non-initial cluster monomials (of a certain acyclic quantum cluster algebra) as the images of rigid objects. Tracing the other path through the tetrahedron gives elements of the quantum shuffle algebra which, according to the dual canonical basis conjecture, should be expected to identify (in symmetric types) with dual canonical basis elements, i.e. with irreducible characters of Khovanov-LaudaRouquier (quiver Hecke) algebras [14, 15, 32, 33] living in the quantum shuffle algebra. In Section 8 we recall the necessary background material to precisely formulate this conjecture as well as sketch a proof for symmetric types giving an alternative to the proof by Kimura and 
Qin using graded quiver varieties. Assuming the conjecture, we formulate a mutation operation for certain clusters defined using the representation theory of KLR algebras. We finish by presenting several conjectures about how this should be done in general.

The results of this work are undoubtedly closely related to several other works in this area though the precise connections are still a little bit of a mystery, perhaps the other constructions provide the categorified/geometric perspective of our constructions. We mention here only a few main examples that should be investigated further. The geometric construction of KLR algebras by Varagnolo-Vasserot [37] and Webster [38] motivate our approach to the dual canonical basis conjecture in acyclic skew-symmetric types, more precisely they have given a geometric construction of certain faithful modules over KLR algebra using flag varieties of quiver representations. The other works we mention are mainly focused on understanding the precise relationship of the representation theory of KLR algebras to an alternative categorification of quantum groups using quantum affine algebras [13]. A central ingredient in establishing a direct link was the paper [9] where the authors find an isomorphism between between a deformed Grothendieck ring associated to the quantum affine algebra and the derived Hall algebra of a quiver [36]. Tracing through these isomorphisms one should find a direct relationship between the derived Hall algebra and the KLR algebra, perhaps a Feigin-type homomorphism is lurking in the background.

The structure of the paper is as follows: Section 2 sets up certain combinatorial and notational conventions that we have found most useful in these investigations. Section 3 recalls the quantized Kac-Moody algebra and background on classical Feigin homomorphisms. In Section 4 we introduce quantum cluster algebras. Section 5 defines valued quivers and their Hall-Ringel algebras and recalls the results of $[28,34,35]$ and [3] relating these to quantum cluster algebras. Sections 6-8 have been discussed above. Section 9 discusses ideas for generalizations and proposes several conjectures on irreducible KLR modules.

\section{Combinatorial conventions and notations}

Fix an indeterminate $q$. We define the $q$-numbers, $q$-factorials, and $q$-binomials by

$$
\begin{aligned}
& (n)=(n)_{q}=1+q+\cdots+q^{n-1}, \\
& (n) !=(n) \cdot(n-1) \cdots(2) \cdot(1), \quad\left(\begin{array}{l}
n \\
k
\end{array}\right)_{q}=\frac{(n) !}{(k) ! \cdot(n-k) !} .
\end{aligned}
$$

In defining our generalized Feigin homomorphism $\bar{\Psi}_{\mathbf{i}}$ we will make particular use of the barinvariant versions of the quantum numbers, for convenience we introduce $v=\sqrt{q}$ :

$$
\begin{aligned}
& {[n]=[n]_{q}=v^{-n+1}+v^{-n+3}+\cdots+v^{n-3}+v^{n-1},} \\
& {[n] !=[n] \cdot[n-1] \cdots[2] \cdot[1], \quad\left[\begin{array}{l}
n \\
k
\end{array}\right]_{q}=\frac{[n] !}{[k] ! \cdot[n-k] !} .}
\end{aligned}
$$

The $q$-binomials satisfy several useful analogues of classical binomial identities. We collect these and sketch their proofs in the following.

\section{Lemma 2.1.}

1. The q-binomial coefficients satisfy the following identities:

(a) Pascal identities: $\left(\begin{array}{l}n \\ k\end{array}\right)_{q}=\left(\begin{array}{c}n-1 \\ k-1\end{array}\right)_{q}+q^{k}\left(\begin{array}{c}n-1 \\ k\end{array}\right)_{q}=q^{n-k}\left(\begin{array}{c}n-1 \\ k-1\end{array}\right)_{q}+\left(\begin{array}{c}n-1 \\ k\end{array}\right)_{q}$;

(b) row identity: for $n>0, \sum_{k=0}^{n}(-1)^{k} q^{-n k+\frac{1}{2} k(k+1)}\left(\begin{array}{l}n \\ k\end{array}\right)_{q}=0$; 
(c) subspace identity: $\left(\begin{array}{c}m+n \\ k\end{array}\right)_{q}=\sum_{r+s=k} q^{r(n-s)}\left(\begin{array}{c}m \\ r\end{array}\right)_{q}\left(\begin{array}{c}n \\ s\end{array}\right)_{q}$.

2. The bar-invariant q-binomial coefficients satisfy the following identities:

(a) Pascal identities: $\left[\begin{array}{l}n \\ k\end{array}\right]_{q}=v^{n-k}\left[\begin{array}{c}n-1 \\ k-1\end{array}\right]_{q}+v^{-k}\left[\begin{array}{c}n-1 \\ k\end{array}\right]_{q}=v^{k-n}\left[\begin{array}{c}n-1 \\ k-1\end{array}\right]_{q}+v^{k}\left[\begin{array}{c}n-1 \\ k\end{array}\right]_{q}$;

(b) row identity: for $n>0, \sum_{k=0}^{n}(-1)^{k} v^{k-n k}\left[\begin{array}{l}n \\ k\end{array}\right]_{q}=0$;

(c) subspace identity: $\left[\begin{array}{c}m+n \\ k\end{array}\right]_{q}=\sum_{r+s=k} v^{r(n-s)-s(m-r)}\left[\begin{array}{c}m \\ r\end{array}\right]_{q}\left[\begin{array}{l}n \\ s\end{array}\right]_{q}$.

Proof. We prove the identities in (1). The Pascal identities in (a) follow from the definition and the equalities $(n)_{q}=(k)_{q}+q^{k}(n-k)_{q}=q^{n-k}(k)_{q}+(n-k)_{q}$. For (b) we simply apply the second Pascal identity to get a telescopic summation:

$$
\begin{aligned}
& \sum_{k=0}^{n}(-1)^{k} q^{-n k+\frac{1}{2} k(k+1)}\left(\begin{array}{l}
n \\
k
\end{array}\right)_{q} \\
& \quad=\sum_{k=1}^{n}(-1)^{k} q^{-n(k-1)+\frac{1}{2}(k-1) k}\left(\begin{array}{l}
n-1 \\
k-1
\end{array}\right)_{q}+\sum_{k=0}^{n-1}(-1)^{k} q^{-n k+\frac{1}{2} k(k+1)}\left(\begin{array}{c}
n-1 \\
k
\end{array}\right)_{q}=0 .
\end{aligned}
$$

To see (c) we consider a finite field $\mathbb{F}$ with $q$ elements. Write $\operatorname{Gr}_{k}\left(\mathbb{F}^{n}\right)$ for the set of $k$-dimensional subspaces of $\mathbb{F}^{n}$. The number of points in $\operatorname{Gr}_{k}\left(\mathbb{F}^{n}\right)$ is given by $\left(\begin{array}{l}n \\ k\end{array}\right)_{q}$. Fix a decomposition $\mathbb{F}^{m+n}=$ $\mathbb{F}^{m} \oplus \mathbb{F}^{n}$ so that any subspace of $\mathbb{F}^{m+n}$ can be decomposed as a direct sum of a subspace of $\mathbb{F}^{m}$ and a subspace of $\mathbb{F}^{n}$. This gives rise to a surjective map $\operatorname{Gr}_{k}\left(\mathbb{F}^{m+n}\right) \rightarrow \bigsqcup_{r+s=k} \operatorname{Gr}_{r}\left(\mathbb{F}^{m}\right) \times \operatorname{Gr}_{s}\left(\mathbb{F}^{n}\right)$ with fiber over any point of $\mathrm{Gr}_{r}\left(\mathbb{F}^{m}\right) \times \mathrm{Gr}_{s}\left(\mathbb{F}^{n}\right)$ an affine space of dimension $r(n-s)$. Now counting points completes the proof when $q$ is a power of a prime. But this is an equality of polynomials for infinitely many values and thus the polynomials must be equal for every $q$.

The identities in (2) easily follow from those in (1) using the relation $\left[\begin{array}{l}n \\ k\end{array}\right]_{q}=v^{-k(n-k)}\left(\begin{array}{l}n \\ k\end{array}\right)_{q}$.

We choose a notation for symmetric groups which is most convenient for describing the structure constants of the multiplication in the quantum shuffle algebra. For each positive integer $t$ let $\Sigma_{t}$ denote the symmetric group on $t$ letters, thought of as the set of all orderings $\sigma=\left(\sigma_{1}, \ldots, \sigma_{t}\right)$ of the set $(1, \ldots, t)$. We write $\sigma \cdot \tau=\left(\sigma_{\tau_{1}}, \ldots, \sigma_{\tau_{t}}\right)$ for the multiplication in $\Sigma_{t}$. Let $\sigma_{k}^{-1}$ denote the position of $k$ in $\sigma$, i.e the number $i$ so that $\sigma_{i}=k$.

For positive integers $r$ and $s$ define the shuffle subgroup $\Sigma_{r, s} \subset \Sigma_{r+s}$ consisting of all shuffles of the sequences $(1, \ldots, r)$ and $(r+1, \ldots, r+s)$, that is

$$
\Sigma_{r, s}=\left\{\sigma \in \Sigma_{r+s}: \sigma_{1}^{-1}<\cdots<\sigma_{r}^{-1}, \sigma_{r+1}^{-1}<\cdots<\sigma_{r+s}^{-1}\right\} .
$$

By convention we take $\Sigma_{0,0}=\Sigma_{0}=\{1\}$ to be the trivial group.

\section{Quantum groups and Feigin homomorphisms}

In this section we introduce the main objects underlying the present investigations: the quantum groups associated to a symmetrizable Cartan matrix and the Feigin homomorphisms mapping them to quantum polynomial algebras.

Fix an index set $I$. Let $A=\left(a_{i j}\right)$ be an $I \times I$ Cartan matrix with symmetrizing matrix $D=\operatorname{diag}(\mathbf{d})$, where $\mathbf{d}=\left(d_{i}: i \in I\right)$ is an $I$-tuple of positive integers. More explicitly, the entries of $A$ have the following properties:

- $a_{i i}=2$ for all $i$; 
- $a_{i j} \leq 0$ for $i \neq j$;

- $d_{i} a_{i j}=d_{j} a_{j i}$ for all $i, j \in I$.

Let $\mathcal{Q}$ denote the root lattice of a Kac-Moody root system $\Phi$ associated to $A$. Denoting by $\left\{\alpha_{i}\right\}_{i \in I}$ the simple roots of $\Phi, \mathcal{Q}$ is the free abelian group with basis $\left\{\alpha_{i}\right\}$. The pair $(A, \mathbf{d})$ determines a symmetric bilinear form $(\cdot, \cdot): \mathcal{Q} \times \mathcal{Q} \rightarrow \mathbb{Z}$ given on generators of $\mathcal{Q}$ by $\left(\alpha_{i}, \alpha_{j}\right)=$ $d_{i} a_{i j}$ for $i, j \in I$. Let $W$ denote the Weyl group associated to $\Phi$ with generators the simple reflections $s_{i}(i \in I)$. Write $\alpha_{i}^{\vee}=\alpha_{i} / d_{i}$. The action of a simple generator $s_{i}$ on a root $\alpha$ is given by

$$
s_{i}(\alpha)=\alpha-\left(\alpha_{i}^{\vee}, \alpha\right) \alpha_{i} .
$$

Notice that $(\cdot, \cdot)$ is equivariant with respect to the action of $W$, i.e. $(\alpha, \beta)=\left(s_{i} \alpha, s_{i} \beta\right)$ for all $\alpha, \beta \in \mathcal{Q}$ and $i \in I$.

Fix an indeterminate $q$ and write $v=\sqrt{q}$. Define $\mathcal{A}_{v}=\mathbb{Z}\left[v^{ \pm 1}\right]$. It will be convenient for $i \in I$ to abbreviate $(n)_{i}=(n)_{q_{i} d_{i}}$ and define $(n)_{i}^{!},\left(\begin{array}{l}n \\ k\end{array}\right)_{i}$ similarly. Similar abbreviations will be used for the bar-invariant versions. Associated to the Cartan matrix $A$ with choice of symmetrizers $\mathbf{d}$ we have the (integral form of the) quantized coordinate ring $\mathcal{A}_{v}[N]$ of the standard upper unipotent subgroup $N$ of the Kac-Moody group $G$. The $\mathcal{Q}$-graded algebra $\mathcal{A}_{v}[N]$ is generated by formal variables $x_{i}^{[r]}\left(i \in I, r \in \mathbb{Z}_{\geq 0}\right)$ with $\left|x_{i}^{[r]}\right|=-r \alpha_{i}$ subject to the quantum Serre relations:

$$
\sum_{r=0}^{1-a_{i j}}(-1)^{r} x_{i}^{[r]} x_{j} x_{i}^{\left[1-a_{i j}-r\right]}=0 \quad \text { for any } i \neq j \in I,
$$

where by definition $[r]_{i}^{!} x_{i}^{[r]}=x_{i}^{r}$. The algebra $\mathcal{A}_{v}[N]$ is actually a twisted bialgebra where each generator is primitive, i.e. the comultiplication is given by $\Delta\left(x_{i}\right)=x_{i} \otimes 1+1 \otimes x_{i}$ for each $i \in I$, and where the multiplication on $\mathcal{A}_{v}[N] \otimes \mathcal{A}_{v}[N]$ is twisted by the grading, i.e. $(x \otimes y)\left(x^{\prime} \otimes y^{\prime}\right)=$ $v^{\left(\left|x^{\prime}\right|,|y|\right)} \cdot x x^{\prime} \otimes y y^{\prime}$.

The graded dual of $\mathcal{A}_{v}[N]$ is the quantized enveloping algebra $U_{v}$ generated by $E_{i}^{[r]}(i \in I$, $r \in \mathbb{Z}_{\geq 0}$ ) also subject to the quantum Serre relations:

$$
\sum_{r=0}^{1-a_{i j}}(-1)^{r} E_{i}^{[r]} E_{j} E_{i}^{\left[1-a_{i j}-r\right]}=0 \quad \text { for any } i \neq j \in I,
$$

where by definition $[r]_{i}^{!} E_{i}^{[r]}=E_{i}^{r}$. The algebra $U_{v}$ is also $\mathcal{Q}$-graded by the rule $\left|E_{i}^{[r]}\right|=r \alpha_{i}$ for $i \in I$.

A powerful tool for studying certain distinguished elements of $\mathcal{A}_{v}[N]$ are the twisted derivations $\theta_{i}, \theta_{i}^{*}: \mathcal{A}_{v}[N] \rightarrow \mathcal{A}_{v}[N]$ which satisfy $\theta_{i}\left(x_{j}\right)=\theta_{i}^{*}\left(x_{j}\right)=\delta_{i j}$ and

$$
\theta_{i}(x y)=v^{\left(\alpha_{i},|y|\right)} \theta_{i}(x) y+x \theta_{i}(y), \quad \theta_{i}^{*}(x y)=\theta_{i}^{*}(x) y+v^{\left(\alpha_{i},|x|\right)} x \theta_{i}^{*}(y) .
$$

It is well known that the maps $\varphi, \varphi^{*}: U_{v} \rightarrow \operatorname{End}\left(\mathcal{A}_{v}[N]\right)$ given by $E_{i} \mapsto \theta_{i}$ or $E_{i} \mapsto \theta_{i}^{*}$ define respectively left and right actions of $U_{v}$ on $\mathcal{A}_{v}[N]$.

Consider a word $\mathbf{i}=\left(i_{1}, \ldots, i_{m}\right) \in I^{m}$ and define the $m$-dimensional quantum polynomial ring

$$
P_{\mathbf{i}}=\mathbb{Z}\left[v^{ \pm \frac{1}{2}}\right]\left\langle t_{1}, \ldots, t_{m}: t_{\ell} t_{k}=v^{\left(\alpha_{i_{k}}, \alpha_{i_{\ell}}\right)} t_{k} t_{\ell} \text { for } k<\ell\right\rangle .
$$

For $\mathbf{a}=\left(a_{1}, \ldots, a_{m}\right) \in \mathbb{Z}^{m}$ define the bar-invariant basis element $t^{\mathbf{a}} \in P_{\mathbf{i}}$ by

$$
t^{\mathbf{a}}=v^{\frac{1}{2} \sum_{k<\ell} a_{k} a_{\ell}\left(\alpha_{i_{k}}, \alpha_{i_{\ell}}\right)} t_{1}^{a_{1}} \cdots t_{m}^{a_{m}} .
$$


Theorem $3.1([2])$. For $\mathbf{i} \in I^{m}$ the map $\Psi_{\mathbf{i}}: \mathcal{A}_{v}[N] \rightarrow P_{\mathbf{i}}$, given on generators by $\Psi_{\mathbf{i}}\left(x_{j}\right)=$ $\sum_{k: i_{k}=j} t_{k}$ for $j \in I$, defines an algebra homomorphism.

We will call $\Psi_{\mathbf{i}}$ the Feigin homomorphism of type $\mathbf{i}$. These homomorphisms were proposed by Boris Feigin (hence the name) as a tool for studying the skew-field of fractions of $\mathcal{A}_{v}[N]$. The Feigin homomorphisms were extensively studied in $[2,10,12]$ where many important properties were discovered. In particular we emphasize the following result.

Theorem $3.2([2])$. Suppose $\mathbf{i} \in I^{m}$ is a reduced word for a Weyl group element $w=s_{i_{1}} \cdots s_{i_{m}} \in W$. Then the kernel $K_{w}:=\operatorname{ker} \Psi_{\mathbf{i}}$ does not depend on the choice of reduced word $\mathbf{i}$ for $w$. Moreover, $K_{w}$ is equal to the structural ideal of the quantized coordinate ring of the closure of the unipotent cell $N^{w}:=N \cap B_{-} w B_{-}$, where $B_{-}$is the standard negative Borel subgroup of $G$, i.e. under the specialization $v \mapsto 1$ the algebra $\mathcal{A}_{v}[N] / K_{w}$ becomes the (integral form of) the coordinate ring of $\overline{N^{w}}$.

The ring $\mathcal{A}_{v}\left[\overline{N^{w}}\right]$ has a natural choice of "coefficients" which become monomials under $\Psi_{\mathbf{i}}$ (see [3, Section 6] for more details), in particular they provide an Ore set by which we may localize to obtain a quantization of the coordinate ring of $N^{w}$. In particular, this shows that the Feigin homomorphism $\Psi_{\mathbf{i}}$ allows one to replace the complicated algebra $\mathcal{A}_{v}\left[N^{w}\right]$ by a relatively simple subalgebra of quantum Laurent polynomials. It turns out that $\Psi_{\mathbf{i}}$ also provides a powerful tool for revealing quantum cluster algebra structures (see the next section for details).

\section{Quantum cluster algebras}

A quantum cluster algebra is a certain type of non-commutative algebra recursively defined from some initial combinatorial data. In this section we recall the combinatorial construction of quantum cluster algebras from an initial seed.

Let $Q$ be an acyclic quiver with vertex set $I$ and recall the symmetrizing matrix $D=\operatorname{diag}(\mathbf{d})$, where $\mathbf{d}=\left(d_{i}: i \in I\right)$. Write $n_{i j}=n_{j i}$ for the number of arrows connecting vertices $i, j \in I$ in $Q$ and note that all such arrows point in the same direction. We assume further that there are only finitely many arrows whose source or target is the vertex $i \in I$. From the pair $(Q, \mathbf{d})$, called a valued quiver, we define an adjacency matrix $B=B_{Q}=\left(b_{i j}\right)$ by the rule

$$
b_{i j}= \begin{cases}n_{i j} d_{j} / \operatorname{gcd}\left(d_{i}, d_{j}\right) & \text { if } i \rightarrow j \text { in } Q \\ -n_{i j} d_{j} / \operatorname{gcd}\left(d_{i}, d_{j}\right) & \text { if } j \rightarrow i \text { in } Q \\ 0 & \text { if } i=j\end{cases}
$$

Notice that the matrix $B$ is skew-symmetrizable with skew-symmetrizing matrix $D$, i.e. $d_{i} b_{i j}=$ $-d_{j} b_{j i}$ for all $i, j \in I$. To connect with the previous section we will assume that the matrix $B$ is related to the Cartan matrix $A$ by $a_{i j}=-\left|b_{i j}\right|$ for $i \neq j$.

Consider an index set $J \supset I$ and a $J \times I$ matrix $\widetilde{B}=\left(b_{i j}\right)$ with principal $I \times I$ submatrix $B$. We say that a skew-symmetric $J \times J$ matrix $\Lambda=\left(\lambda_{i j}\right)$ is compatible with $\widetilde{B}$ if

$$
\sum_{i \in J} \lambda_{i j} b_{i k}=\delta_{j k} d_{k} \quad \text { for all } j \in J \text { and } k \in I .
$$

Associated to $\Lambda$ we have the quantum torus $\mathcal{T}_{\Lambda, q}$ generated over $\mathbb{Z}\left[v^{ \pm 1}\right]$ by quasi-commuting cluster variables $\mathbf{X}=\left(X_{i}: i \in J\right)$ subject to the relations

$$
X_{i} X_{j}=q^{\lambda_{i j}} X_{j} X_{i} \quad \text { for } i, j \in J .
$$

We will call the pair $\Sigma_{0}=(\mathbf{X}, \widetilde{B})$ a quantum seed whenever $\Lambda$ is compatible with $\widetilde{B}$, the collection $\mathbf{X}$ is called the cluster and $\widetilde{B}$ is called the exchange matrix. 
Remark 4.1. It is often customary to include the commutation matrix $\Lambda$ in the data of a quantum seed, however the quantum cluster $\mathbf{X}$ "knows" its quasi-commutation so this is slightly redundant and we omit it.

In order to define the mutation of quantum seeds we need to introduce more notation. Choose a total order $<$ on $J$. Then for $\mathbf{a} \in \mathbb{Z}^{J}$ we may define the bar-invariant monomial $X^{\mathbf{a}} \in \mathcal{T}_{\Lambda, q}$ by

$$
X^{\mathbf{a}}=v^{\sum_{i<j} a_{i} a_{j} \lambda_{j i}} \vec{\prod}_{i \in J} X_{i}^{a_{i}}
$$

where $\vec{\prod}$ denotes the product in increasing order. Then for $k \in I$ we are now ready to define the mutation in direction $k, \mu_{k} \Sigma=\left(\mu_{k} \mathbf{X}, \mu_{k} \widetilde{B}\right)$, as follows:

- $\mu_{k} \mathbf{X}=\mathbf{X} \backslash\left\{X_{k}\right\} \cup\left\{X_{k}^{\prime}\right\}$ for $X_{k}^{\prime}$ given by

$$
X_{k}^{\prime}=X^{\mathbf{b}_{+}^{k}-\alpha_{k}}+X^{\mathbf{b}_{-}^{k}-\alpha_{k}},
$$

where $\mathbf{b}_{+}^{k}, \mathbf{b}_{-}^{k} \in \mathbb{Z}_{\geq 0}^{J}$ are the unique vectors satisfying $\mathbf{b}_{+}^{k}-\mathbf{b}_{-}^{k}=\mathbf{b}^{k}$ is the $k^{\text {th }}$ column of $\widetilde{B}$;

- $\mu_{k} \widetilde{B}=\left(b_{i j}^{\prime}\right)$, where

$$
b_{i j}^{\prime}= \begin{cases}-b_{i j} & \text { if } i=k \text { or } j=k, \\ b_{i j}+\left[b_{i k}\right]_{+} b_{k j}+b_{i k}\left[-b_{k j}\right]_{+} & \text {otherwise. }\end{cases}
$$

Note that the compatibility of $\tilde{B}$ and $\Lambda$ ensures that $\mu_{k} \mathbf{X}$ is again a quantum cluster. Precise formulas for the commutation matrix of $\mu_{k} \mathbf{X}$ are known [4], however we will not need them.

Now consider a rooted, labelled $I$-regular tree $\mathbb{T}$ with root vertex $t_{0}$, where the edges emanating from each vertex are labelled by distinct elements of $I$. We assign quantum seeds $\Sigma_{t}=\left(\mathbf{X}_{t}, \widetilde{B}_{t}\right)$ to the vertices $t \in \mathbb{T}$ so that $\Sigma_{t_{0}}=\Sigma_{0}$ and for $t \underline{k} t^{\prime}$ in $\mathbb{T}$ the seeds $\Sigma_{t}$ and $\Sigma_{t^{\prime}}$ are related by the mutation in direction $k$. For any two (possibly distant) vertices $t, t^{\prime} \in \mathbb{T}$ we say that the seeds $\Sigma_{t}$ and $\Sigma_{t^{\prime}}$ are mutation equivalent. A fundamental result in the theory of quantum cluster algebras is the following.

Theorem 4.2 ([4, quantum Laurent phenomenon]). For any vertex $t \in \mathbb{T}$ all cluster variables of the cluster $\mathbf{X}_{t}$ are contained in $\mathcal{T}_{\Lambda, q}$.

The quantum cluster algebra is then the $\mathbb{Z}\left[v^{ \pm 1}\right]\left[X_{j}^{ \pm 1}: j \in J \backslash I\right]$-subalgebra of $\mathcal{T}_{\Lambda, q}$ generated by all cluster variables from all seeds mutation equivalent to $\Sigma_{0}$ :

$$
\mathcal{A}_{q}(\mathbf{X}, \widetilde{B})=\mathbb{Z}\left[v^{ \pm 1}\right]\left[X_{j}^{ \pm 1}: j \in J \backslash I\right]\left[X_{i ; t}: t \in \mathbb{T}, i \in I\right] \subset \mathcal{T}_{\Lambda, q}
$$

One of the main problems in the theory of quantum cluster algebras is to explicitly describe the Laurent expansions of all cluster variables. In general this is still an open and active area of research. Under the current assumption of acyclicity some progress has been made on this problem using the representation theory of valued quivers; we present these results at the beginning of the next section.

\section{Representations of valued quivers and their Hall-Ringel algebras}

Fix a finite field $\mathbb{F}$ and an algebraic closure $\overline{\mathbb{F}}$. For each positive integer $d$ write $\mathbb{F}_{d}$ for the degree $d$ extension of $\mathbb{F}$ in $\overline{\mathbb{F}}$. Note that with these conventions we may intersect $\mathbb{F}_{d}$ and $\mathbb{F}_{d^{\prime}}$ to 
get that $\mathbb{F}_{\operatorname{gcd}\left(d, d^{\prime}\right)}$ is their largest common subfield. We now define representations of $(Q, \mathbf{d})$ by assigning an $\mathbb{F}_{d_{i}}$-vector space to each vertex $i \in I$ and an $\mathbb{F}_{\operatorname{gcd}\left(d_{i}, d_{j}\right)}$-linear map to each arrow from vertex $i$ to vertex $j$. The finite-dimensional representations of $(Q, \mathbf{d})$ form a finitary, hereditary, Abelian category $\operatorname{rep}_{\mathbb{F}}(Q, \mathbf{d})$ where kernels and cokernels are taken vertex-wise, we refer the reader to [34] for more details.

Recall that we work under the assumption that the quiver $Q$ contains no oriented cycles. Then the simple representations of $(Q, \mathbf{d})$ may be labeled as $S_{i}(i \in I)$ where the only non-zero vector space is $\mathbb{F}_{d_{i}}$ at vertex $i$. The Grothendieck group $\mathcal{K}(Q, \mathbf{d})$ of $\operatorname{rep}_{\mathbb{F}}(Q, \mathbf{d})$ has a $\mathbb{Z}$-basis given by the classes $\alpha_{i}=\left[S_{i}\right](i \in I)$ of the irreducible representations $S_{i}$, in this way we identify the root lattice $\mathcal{Q}$ with $\mathcal{K}(Q, \mathbf{d})$. Write $[V]$ for the isomorphism class of a representation $V \in \operatorname{rep}_{\mathbb{F}}(Q, \mathbf{d})$ and write $|V|$ for its dimension vector, i.e. the class of $V$ in $\mathcal{K}(Q, \mathbf{d})$. Define the height $\operatorname{ht}(V)$ of a representation $V$ as the length of any composition series for $V$.

We define the Euler-Ringel form $\langle\cdot, \cdot\rangle: \mathcal{K}(Q, \mathbf{d}) \times \mathcal{K}(Q, \mathbf{d}) \rightarrow \mathbb{Z}$ on generators by

$$
\left\langle\alpha_{i}, \alpha_{j}\right\rangle= \begin{cases}d_{i} & \text { if } i=j, \\ -\left[d_{i} b_{i j}\right]_{+} & \text {if } i \neq j .\end{cases}
$$

The identification of $\mathcal{Q}$ and $\mathcal{K}(Q, \mathbf{d})$ allows to transfer the bilinear form $(\cdot, \cdot)$ to $\mathcal{K}(Q, \mathbf{d})$. It then follows from the definitions that $(\cdot, \cdot)$ is the symmetrization of the Euler-Ringel form, i.e. $\left(\alpha_{i}, \alpha_{j}\right)=\left\langle\alpha_{i}, \alpha_{j}\right\rangle+\left\langle\alpha_{j}, \alpha_{i}\right\rangle$ for all $i, j \in I$. Recall that we write $\alpha_{i}^{\vee}=\alpha_{i} / d_{i}$. For a dimension vector $\mathbf{v} \in \mathcal{K}(Q, \mathbf{d})$ define ${ }^{*} \mathbf{v} \in \mathcal{K}(Q, \mathbf{d})$ by

$$
{ }^{*} \mathbf{v}=\sum_{i \in I}\left\langle\alpha_{i}^{\vee}, \mathbf{v}\right\rangle \alpha_{i}
$$

In [34] we proposed the following definition and established the additive categorification of Theorem 5.2 below in a restricted set of cases.

Definition 5.1. For any representation $V \in \operatorname{rep}_{\mathbb{F}}(Q, \mathbf{d})$ we associate the quantum cluster character $X_{V}$ defined by

$$
X_{V}=\sum_{\mathbf{e} \in \mathcal{K}(Q, \mathbf{d})}|\mathbb{F}|^{-\frac{1}{2}\langle\mathbf{e},|V|-\mathbf{e}\rangle} \cdot\left|\operatorname{Gr}_{\mathbf{e}}(V)\right| \cdot X^{-\widetilde{B} \mathbf{e}-{ }^{*}|V|} \in \mathcal{T}_{\Lambda,|\mathbb{F}|},
$$

where $\operatorname{Gr}_{\mathbf{e}}(V)$ denotes the set of subrepresentations of $V$ with dimension vector $\mathbf{e}$.

A representation $V$ is rigid if $\operatorname{Ext}^{1}(V, V)=0$. Our main result from [35] is the following. We also refer the reader to [28] for an analogous result in the equally valued case, i.e. when $d_{i}=1$ for all $i \in I$.

Theorem 5.2 ([28, 35]). Every non-initial cluster variable of $\mathcal{A}_{|\mathbb{F}|}(\mathbf{X}, \widetilde{B})$ is of the form $X_{V}$ for some indecomposable rigid representation $V$ of $(Q, \mathbf{d})$.

The multiplication formulas for quantum cluster characters involved in the proof of Theorem 5.2 suggest a relationship with dual Hall-Ringel algebras. In the work [3] we studied with Arkady Berenstein the nature of this relationship, a review of these results are presented in the next section.

\subsection{Generalized Feigin homomorphisms}

Define the Hall-Ringel bialgebra $\mathcal{H}(Q, \mathbf{d})=\bigoplus \mathbb{C}[V]$ to be the free $\mathcal{K}(Q, \mathbf{d})$-graded $\mathbb{C}$-vector space spanned by the isomorphism classes of representations of $(Q, \mathbf{d})$ with grading $|[V]|=|V|$ given by dimension vector. The structure constants of the multiplication and comultiplication 
count certain extensions between representations. More precisely, for representations $U, V$, and $W$, we define the Hall number as the cardinality of the following set:

$$
\mathcal{F}_{U W}^{V}=\{R \subset V: R \cong W \text { and } V / R \cong U\} .
$$

For convenience we abbreviate $v=\sqrt{|\mathbb{F}|}$.

Theorem $5.3([29])$. The map $\mu: \mathcal{H}(Q, \mathbf{d}) \otimes \mathcal{H}(Q, \mathbf{d}) \rightarrow \mathcal{H}(Q, \mathbf{d})$ (written multiplicatively) given on basis elements by

$$
[U][W]=\sum_{[V]} v^{\langle|U|,|W|\rangle}\left|\mathcal{F}_{U W}^{V}\right| \cdot[V]
$$

defines an associative multiplication on $\mathcal{H}(Q, \mathbf{d})$.

The tensor square $\mathcal{H}(Q, \mathbf{d}) \otimes \mathcal{H}(Q, \mathbf{d})$ will be considered as an algebra via the twisted multiplication defined by

$$
([U] \otimes[W])\left(\left[U^{\prime}\right] \otimes\left[W^{\prime}\right]\right)=v^{\left(|W|,\left|U^{\prime}\right|\right)}[U]\left[U^{\prime}\right] \otimes[W]\left[W^{\prime}\right] .
$$

Theorem $5.4([7])$. The map $\Delta: \mathcal{H}(Q, \mathbf{d}) \rightarrow \mathcal{H}(Q, \mathbf{d}) \otimes \mathcal{H}(Q, \mathbf{d})$ given on basis elements by

$$
\Delta([V])=\sum_{[U],[W]} v^{\langle|U|,|W|\rangle} \frac{|\operatorname{Aut}(U)||\operatorname{Aut}(W)|}{|\operatorname{Aut}(V)|} \cdot\left|\mathcal{F}_{U W}^{V}\right| \cdot[U] \otimes[W]
$$

defines a coassociative comultiplication on $\mathcal{H}(Q, \mathbf{d})$ which is compatible with the multiplication on $\mathcal{H}(Q, \mathbf{d})$ and the twisted multiplication on $\mathcal{H}(Q, \mathbf{d}) \otimes \mathcal{H}(Q, \mathbf{d})$.

The graded dual Hall-Ringel bialgebra $\mathcal{H}^{*}(Q, \mathbf{d})$ has a basis of delta functions $\delta_{[V]}$ with grading $\left|\delta_{[V]}\right|=-|V|$. It will be convenient to consider a slight rescaling $[V]^{*}$ of the standard dual basis given by

$$
[V]^{*}=v^{-\frac{1}{2}\langle V, V\rangle+\frac{1}{2} \sum_{i=1}^{n} d_{i} v_{i}} \delta_{[V]}
$$

For a representation $U \in \operatorname{rep}_{\mathbb{F}}(Q, \mathbf{d})$ define the linear maps $\theta_{U}, \theta_{U}^{*}: \mathcal{H}^{*}(Q, \mathbf{d}) \rightarrow \mathcal{H}^{*}(Q, \mathbf{d})$ by

$$
\theta_{U}\left(\delta_{[V]}\right)([W])=\delta_{[V]}([W][U]) \quad \text { and } \quad \theta_{U}^{*}\left(\delta_{[V]}\right)([W])=\delta_{[V]}([U][W])
$$

for each $V, W \in \operatorname{rep}_{\mathbb{F}}(Q, \mathbf{d})$. More precisely, we may expand $\theta_{U}\left(\delta_{[V]}\right)$ and $\theta_{U}^{*}\left(\delta_{[V]}\right)$ in the basis of delta functions as follows:

$$
\theta_{U}\left(\delta_{[V]}\right)=\sum_{[W]} v^{\langle|W|,|U|\rangle}\left|\mathcal{F}_{W U}^{V}\right| \cdot \delta_{[W]}, \quad \theta_{U}^{*}\left(\delta_{[V]}\right)=\sum_{[W]} v^{\langle|U|,|W|\rangle}\left|\mathcal{F}_{U W}^{V}\right| \cdot \delta_{[W]}
$$

The following result asserts that for any $i \in I$ the linear maps $\theta_{S_{i}}$ and $\theta_{S_{i}}^{*}$ are twisted derivations on $\mathcal{H}^{*}(Q, \mathbf{d})$.

Lemma 5.5. For any simple representation $S_{i}$ of $(Q, \mathbf{d})$ and $U, W \in \operatorname{rep}_{\mathbb{F}}(Q, \mathbf{d})$ we have:

$$
\begin{aligned}
& \theta_{S_{i}}\left(\delta_{[U]} \delta_{[W]}\right)=v^{\left(\alpha_{i},|W|\right)} \theta_{S_{i}}\left(\delta_{[U]}\right) \delta_{[W]}+\delta_{[U]} \theta_{S_{i}}\left(\delta_{[W]}\right), \\
& \theta_{S_{i}}^{*}\left(\delta_{[U]} \delta_{[W]}\right)=\theta_{S_{i}}^{*}\left(\delta_{[U]}\right) \delta_{[W]}+v^{\left(\alpha_{i},|U|\right)} \delta_{[U]} \theta_{S_{i}}^{*}\left(\delta_{[W]}\right) .
\end{aligned}
$$


Proof. These easily follow from the description (5.1) of $\theta_{S_{i}}$ and $\theta_{S_{i}}^{*}$. Indeed, for any $V \in$ $\operatorname{rep}_{\mathbb{F}}(Q, \mathbf{d})$ we have

$$
\begin{aligned}
\theta_{S_{i}}\left(\delta_{[U]} \delta_{[W]}\right)([V]) & =\left(\delta_{[U]} \delta_{[W]}\right)\left([V]\left[S_{i}\right]\right)=\left(\delta_{[U]} \otimes \delta_{[W]}\right)\left(\Delta\left([V]\left[S_{i}\right]\right)\right) \\
& =\left(\delta_{[U]} \otimes \delta_{[W]}\right)\left(\Delta([V]) \Delta\left(\left[S_{i}\right]\right)\right) \\
& =\left(\delta_{[U]} \otimes \delta_{[W]}\right)\left(\Delta([V])\left(\left[S_{i}\right] \otimes 1\right)\right)+\left(\delta_{[U]} \otimes \delta_{[W]}\right)\left(\Delta([V])\left(1 \otimes\left[S_{i}\right]\right)\right) .
\end{aligned}
$$

But notice that the twisted multiplication on $\mathcal{H}(Q, \mathbf{d}) \otimes \mathcal{H}(Q, \mathbf{d})$ gives

$$
\left(\delta_{[U]} \otimes \delta_{[W]}\right)\left(\Delta([V])\left(\left[S_{i}\right] \otimes 1\right)\right)=v^{\left(\alpha_{i},|W|\right)}\left(\theta_{S_{1}}\left(\delta_{[U]}\right) \otimes \delta_{[W]}\right)(\Delta([V]))
$$

and that

$$
\left(\delta_{[U]} \otimes \delta_{[W]}\right)\left(\Delta([V])\left(1 \otimes\left[S_{i}\right]\right)\right)=\left(\delta_{[U]} \otimes \theta_{S_{1}}\left(\delta_{[W]}\right)\right)(\Delta([V])) .
$$

Combining these observations completes the proof of the first identity:

$$
\begin{aligned}
\theta_{S_{i}}\left(\delta_{[U]} \delta_{[W]}\right)([V]) & =v^{\left(\alpha_{i},|W|\right)}\left(\theta_{S_{1}}\left(\delta_{[U]}\right) \otimes \delta_{[W]}\right)(\Delta([V]))+\left(\delta_{[U]} \otimes \theta_{S_{1}}\left(\delta_{[W]}\right)\right)(\Delta([V])) \\
& =\left(v^{\left(\alpha_{i},|W|\right)} \theta_{S_{i}}\left(\delta_{[U]}\right) \delta_{[W]}+\delta_{[U]} \theta_{S_{i}}\left(\delta_{[W]}\right)\right)([V]) .
\end{aligned}
$$

The second identity follows by a similar calculation.

Remark 5.6. In general, one cannot hope for $\theta_{U}$ and $\theta_{U}^{*}$ to be derivations. Under certain restrictions one can show that they are higher order differential operators on $\mathcal{H}^{*}(Q, \mathbf{d})$, though we will not use this here and thus leave the details for another day.

For each representation $V \in \operatorname{rep}_{\mathbb{F}}(Q, \mathbf{d})$ we define the quantum $\mathbf{i}$-character

$$
X_{V, \mathbf{i}}=\sum_{\mathbf{a} \in \mathbb{Z}_{\geq 0}^{m}} v^{-\sum_{k<\ell} a_{k} a_{\ell}\left\langle\alpha_{i_{\ell}}, \alpha_{i_{k}}\right\rangle} \cdot\left|\mathcal{F}_{\mathbf{i}, \mathbf{a}}(V)\right| \cdot t^{\mathbf{a}} \in P_{\mathbf{i}},
$$

where $\mathcal{F}_{\mathbf{i}, \mathbf{a}}(V)$ denotes the set of all flags of $V$ of type $(\mathbf{i}, \mathbf{a})$, i.e.

$$
\mathcal{F}_{\mathbf{i}, \mathbf{a}}(V)=\left\{0=V_{m} \subset V_{m-1} \subset \cdots \subset V_{1} \subset V_{0}=V: V_{k-1} / V_{k} \cong S_{i_{k}}^{a_{k}}, 1 \leq k \leq m\right\} .
$$

Theorem $5.7([3])$. For any sequence $\mathbf{i}$ the assignment $[V]^{*} \mapsto X_{V, \mathbf{i}}$ defines an algebra homomorphism $\widetilde{\Psi}_{\mathbf{i}}: \mathcal{H}^{*}(Q, \mathbf{d}) \rightarrow P_{\mathbf{i}}$.

It is a classical result of Ringel [29] that the composition subalgebra of $\mathcal{H}^{*}(Q, \mathbf{d})$ generated over $\mathcal{A}_{v}=\mathbb{Z}\left[v^{ \pm 1}\right]$ by the classes $\left[S_{j}\right]^{*}$ of simple representations is isomorphic to $\mathcal{A}_{v}[N]$. Since $\tilde{\Psi}\left(\left[S_{j}\right]^{*}\right)=\sum_{k: i_{k}=j} t_{k}$ we see that the restriction of $\widetilde{\Psi}_{\mathbf{i}}$ to $\mathcal{A}_{v}[N]$ recovers the Feigin homomorphism $\Psi_{\mathbf{i}}$ introduced in Section 3. Thus we call the maps $\widetilde{\Psi}_{\mathbf{i}}$ generalized Feigin homomorphisms.

A certain choice of generalized Feigin homomorphism realizes the connection between acyclic quantum cluster characters and dual Hall-Ringel algebras alluded to above.

Theorem 5.8 ([3]). Let $\mathbf{i}$ be twice a complete source adapted sequence for $Q$. Then there exists a compatible pair $\left(\widetilde{B}_{\mathbf{i}}, \Lambda_{\mathbf{i}}\right)$ so that $P_{\mathbf{i}} \cong \mathcal{T}_{\Lambda_{\mathbf{i}}, q}$ and $\widetilde{\Psi}_{\mathbf{i}}\left([V]^{*}\right)=X_{V}$ for any representation $V \in \operatorname{rep}_{\mathbb{F}}(Q, \mathbf{d})$.

Combining Theorems 5.2 and 5.8 we see that non-initial cluster variables can be recovered as images of rigid representations under the generalized Feigin homomorphism.

Theorem 5.9 ([3]). Let $c$ be any Coxeter element of $W$ and $\mathbf{i}_{0}$ a reduced word for $c$. The quantized coordinate ring $\mathcal{A}_{v}\left[N^{c^{2}}\right]$ admits the structure of an acyclic quantum cluster algebra associated to the compatible pair $\left(\widetilde{B}_{\mathbf{i}}, \Lambda_{\mathbf{i}}\right)$ for $\mathbf{i}=\left(\mathbf{i}_{0}, \mathbf{i}_{0}\right)$.

Together these results motivate our approach to the dual canonical basis conjecture, we will make this precise in future sections. 


\section{Quantum shuffle algebras}

The quantized coordinate ring $\mathcal{A}_{v}[N]$ admits an embedding into a quantum shuffle algebra associated to the $I \times I$ symmetrizable Cartan matrix $A$ with symmetrizers $\mathbf{d}$. In this section we introduce our choice of quantum shuffle algebra and prove some basic properties. Better proofs of many of these results can be found in [23]. We refer the reader also to [31] for a more general discussion of quantum shuffle algebras.

Let $\mathbf{W}=\bigsqcup_{m \geq 0} I^{m}$ denote the set of all words in the alphabet $I$. For a word $\mathbf{i}=\left(i_{1}, \ldots, i_{m}\right)$ and a sequence of nonnegative integers $\mathbf{c} \in \mathbb{Z}_{\geq 0}^{m}$ we will make use of the following simplifying notation:

$$
\mathbf{i}^{\mathbf{c}}=\left(i_{1}^{c_{1}}, \ldots, i_{m}^{c_{m}}\right)=(\underbrace{i_{1}, \ldots, i_{1}}_{c_{1}}, \ldots, \underbrace{i_{m}, \ldots, i_{m}}_{c_{m}})
$$

where $i_{k}$ appears $c_{k}$ times $(1 \leq k \leq m)$. For $\mathbf{j}=\left(j_{1}, \ldots, j_{r}\right) \in \mathbf{W}$ we will write $|\mathbf{j}|=\alpha_{j_{1}}+\cdots+\alpha_{j_{r}}$ for the degree of the word $\mathbf{j}$. Denote by $\mathbf{W}^{\nu}$ the set of all words of degree $\nu \in \mathcal{Q}$.

Define the quantum shuffle algebra $g^{*}$ as the $\mathbb{Z}\left[v^{ \pm \frac{1}{2}}\right]$-algebra with basis $\mathbf{W}$ and multiplication given by

$$
\left(j_{1}, \ldots, j_{r}\right) \circ\left(j_{r+1}, \ldots, j_{r+s}\right)=\sum_{\sigma \in \Sigma_{r, s}} v^{\zeta(\sigma)} \mathbf{j}_{\sigma}
$$

where $\mathbf{j}_{\sigma}=\left(j_{\sigma_{1}}, \ldots, j_{\sigma_{r+s}}\right)$ and $\zeta(\sigma)=\zeta_{r, s}(\sigma)$ measures both inversions and non-inversions occurring in $\sigma$ :

$$
\zeta(\sigma)=\frac{1}{2} \sum_{\substack{1 \leq k \leq r \\ r+1 \leq \ell \leq r+s \\ \sigma_{\ell}^{-1}<\sigma_{k}^{-1}}}\left(\alpha_{j_{k}}, \alpha_{j_{\ell}}\right)-\frac{1}{2} \sum_{\substack{1 \leq k \leq r \\ r+1 \leq \ell \leq r+s \\ \sigma_{k}^{-1}<\sigma_{\ell}^{-1}}}\left(\alpha_{j_{k}}, \alpha_{j_{\ell}}\right) .
$$

Remark 6.1. Kleshchev-Ram [18] and Leclerc [23] use opposite multiplications for their quantum shuffle algebras measuring only inversions and only non-inversions respectively. The conventions of [18] are claimed to be more convenient for the relationship with the representation theory of quiver Hecke algebras.

We hope that our choice of multiplication on $g^{*}$ will prove equally useful in this regard since this is our preferred quantum shuffle multiplication which will figure prominently in the results presented in this note.

The following proposition can be obtained from the results of [23] by rescaling the basis, however for completeness we provide a proof.

Proposition 6.2. The shuffle product (6.1) defines an associative algebra structure on $g^{*}$.

Proof. It will be convenient to introduce the subgroup $\Sigma_{r, s, t}$ of $\Sigma_{r+s+t}$ consisting of all shuffles of the sequences $(1, \ldots, r),(r+1, \ldots, r+s)$, and $(r+s+1, \ldots, r+s+t)$, that is

$$
\Sigma_{r, s, t}=\left\{\sigma \in \Sigma_{r+s+t}: \sigma_{1}^{-1}<\cdots<\sigma_{r}^{-1}, \sigma_{r+1}^{-1}<\cdots<\sigma_{r+s}^{-1}, \sigma_{r+s+1}^{-1}<\cdots<\sigma_{r+s+t}^{-1}\right\} .
$$

There are natural embeddings $\Sigma_{r, s} \hookrightarrow \Sigma_{r, s, t}$ written $\sigma \mapsto \widetilde{\sigma}$ and $\Sigma_{s, t} \hookrightarrow \Sigma_{r, s, t}$ written $\sigma \mapsto \widetilde{\sigma}^{\prime}$, where

$$
\begin{aligned}
& \tilde{\sigma}_{k}=\sigma_{k} \quad \text { for } \quad 1 \leq k \leq r+s \quad \text { and } \quad \tilde{\sigma}_{r+s+k}=r+s+k \quad \text { for } \quad 1 \leq k \leq t, \\
& \tilde{\sigma}_{k}^{\prime}=k \quad \text { for } \quad 1 \leq k \leq r \quad \text { and } \quad \tilde{\sigma}_{r+k}^{\prime}=r+\sigma_{k} \quad \text { for } \quad 1 \leq k \leq s+t .
\end{aligned}
$$


Notice that every element of $\Sigma_{r, s, t}$ has a unique presentation as $\widetilde{\sigma} \cdot \tau$ with $\sigma \in \Sigma_{r, s}$ and $\tau \in \Sigma_{r+s, t}$ and a unique presentation as $\widetilde{\sigma}^{\prime} \cdot \rho$ with $\sigma \in \Sigma_{s, t}$ and $\rho \in \Sigma_{r, s+t}$. For $\sigma \in \Sigma_{r, s, t}$ define

$$
\begin{aligned}
& \widetilde{\zeta}(\sigma)= \frac{1}{2} \sum_{\substack{1 \leq k \leq r \\
r+1 \leq \ell \leq r+s}}\left(\alpha_{j_{k}}, \alpha_{j_{\ell}}\right)+\frac{1}{2} \sum_{\substack{\sigma_{\ell}^{-1}<\sigma_{k}^{-1} \\
1 \leq k \leq r \\
r+s+1 \leq \ell \leq r+s+t \\
\sigma_{\ell}^{-1}<\sigma_{k}^{-1}}}\left(\alpha_{j_{k}}, \alpha_{j_{\ell}}\right)+\frac{1}{2} \sum_{\substack{r+1 \leq k \leq r+s \\
r+s+1 \leq \ell \leq r+s+t \\
\sigma_{\ell}^{-1}<\sigma_{k}^{-1}}}\left(\alpha_{j_{k}}, \alpha_{j_{\ell}}\right) \\
&-\frac{1}{2} \sum_{\substack{1 \leq k \leq r \\
r+1 \leq \ell \leq r+s \\
\sigma_{k}^{-1}<\sigma_{\ell}^{-1}}}\left(\alpha_{j_{k}}, \alpha_{j_{\ell}}\right)-\frac{1}{2} \sum_{\substack{1 \leq k \leq r \\
r+s+1 \leq \ell \leq r+s+t \\
\sigma_{k}^{-1}<\sigma_{\ell}^{-1}}}\left(\alpha_{j_{k}}, \alpha_{j_{\ell}}\right)-\frac{1}{2} \sum_{\substack{r+1 \leq k \leq r+s \\
r+s+1 \leq \ell \leq r+s+t \\
\sigma_{k}^{-1}<\sigma_{\ell}^{-1}}}\left(\alpha_{j_{k}}, \alpha_{j_{\ell}}\right) .
\end{aligned}
$$

Then one immediately checks that:

$$
\begin{array}{ll}
\widetilde{\zeta}(\widetilde{\sigma} \cdot \tau)=\zeta(\sigma)+\zeta(\tau) & \text { for any } \sigma \in \Sigma_{r, s} \text { and } \tau \in \Sigma_{r+s, t}, \\
\widetilde{\zeta}\left(\widetilde{\sigma}^{\prime} \cdot \rho\right)=\zeta(\sigma)+\zeta(\rho) & \text { for any } \sigma \in \Sigma_{s, t} \text { and } \rho \in \Sigma_{r, s+t} .
\end{array}
$$

We are now ready to prove the proposition. Indeed, on the one hand we have

$$
\begin{aligned}
& \left(\left(j_{1}, \ldots, j_{r}\right) \circ\left(j_{r+1}, \ldots, j_{r+s}\right)\right) \circ\left(j_{r+s+1}, \ldots, j_{r+s+t}\right) \\
& \quad=\sum_{\sigma \in \Sigma_{r, s}} v^{\zeta(\sigma)} \mathbf{j}_{\sigma} \circ\left(j_{r+s+1}, \ldots, j_{r+s+t}\right)=\sum_{\substack{\sigma \in \Sigma_{r, s} \\
\tau \in \Sigma_{r+s, t}}} v^{\zeta(\sigma)+\zeta(\tau)} \mathbf{j}_{\widetilde{\sigma} \cdot \tau}
\end{aligned}
$$

and on the other hand we have

$$
\begin{aligned}
& \left(j_{1}, \ldots, j_{r}\right) \circ\left(\left(j_{r+1}, \ldots, j_{r+s}\right) \circ\left(j_{r+s+1}, \ldots, j_{r+s+t}\right)\right) \\
& \quad=\sum_{\sigma \in \Sigma_{s, t}} v^{\zeta(\sigma)}\left(j_{1}, \ldots, j_{r}\right) \circ \mathbf{j}_{\sigma}=\sum_{\substack{\sigma \in \Sigma_{s, t} \\
\rho \in \Sigma_{r, s+t}}} v^{\zeta(\sigma)+\zeta(\rho)} \mathbf{j}_{\widetilde{\sigma}^{\prime} \cdot \rho} .
\end{aligned}
$$

But both of these expressions are equal to $\sum_{\sigma \in \Sigma_{r, s, t}} v^{\widetilde{\zeta}(\sigma)} \mathbf{j}_{\sigma}$.

We will also need a comultiplication on $g^{*}$. This is defined on a word $\mathbf{h} \in \mathbf{W}$ by

$$
\Delta(\mathbf{h})=\sum_{\mathbf{j}_{1}, \mathbf{j}_{2} \in \mathbf{W}:\left(\mathbf{j}_{1}, \mathbf{j}_{2}\right)=\mathbf{h}} v^{\frac{1}{2}\left(\left|\mathbf{j}_{1}\right|,\left|\mathbf{j}_{2}\right|\right)} \mathbf{j}_{1} \otimes \mathbf{j}_{2}
$$

In the following result we consider $g^{*} \otimes g^{*}$ with the twisted multiplication

$$
\left(\mathbf{j}_{1,1} \otimes \mathbf{j}_{1,2}\right) \circ\left(\mathbf{j}_{2,1} \otimes \mathbf{j}_{2,2}\right)=v^{\left(\left|\mathbf{j}_{1,2}\right|,\left|\mathbf{j}_{2,1}\right|\right)}\left(\mathbf{j}_{1,1} \circ \mathbf{j}_{2,1}\right) \otimes\left(\mathbf{j}_{1,2} \circ \mathbf{j}_{2,2}\right)
$$

for all words $\mathbf{j}_{1,1}, \mathbf{j}_{1,2}, \mathbf{j}_{2,1}, \mathbf{j}_{2,2} \in \mathbf{W}$.

Lemma 6.3. The map $\Delta: g^{*} \rightarrow g^{*} \otimes g^{*}$ defines a coassociative comultiplication on $g^{*}$. Moreover, $g^{*}$ is a bialgebra with counit $\epsilon: g^{*} \rightarrow \mathbb{C}$ given by $\varepsilon(\mathbf{j})=0$ whenever $\mathbf{j} \neq()$ is not the empty word and $\varepsilon(())=1$.

Proof. We will check explicitly the coassociativity and the compatibility of multiplication and comultiplication on $g^{*}$, the other axioms of a bialgebra structure on $g^{*}$ are straightforward and left to the reader.

We start with the coassociativity of $\Delta$. For a word $\mathbf{h} \in \mathbf{W}$ we have

$$
(\Delta \otimes \mathrm{Id}) \Delta(\mathbf{h})=\sum_{\mathbf{j}_{1}, \mathbf{j}_{2} \in \mathbf{W}:\left(\mathbf{j}_{1}, \mathbf{j}_{2}\right)=\mathbf{h}} v^{\frac{1}{2}\left(\left|\mathbf{j}_{1}\right|,\left|\mathbf{j}_{2}\right|\right)} \Delta\left(\mathbf{j}_{1}\right) \otimes \mathbf{j}_{2}
$$




$$
\begin{aligned}
& =\sum_{\substack{\mathbf{j}_{1}, \mathbf{j}_{2} \in \mathbf{W}: \\
\left(\mathbf{j}_{1}, \mathbf{j}_{2}\right)=\mathbf{h}}} v^{\frac{1}{2}\left(\left|\mathbf{j}_{1}\right|,\left|\mathbf{j}_{2}\right|\right)} \sum_{\substack{\mathbf{j}_{1,1}, \mathbf{j}_{1,2} \in \mathbf{W}: \\
\left(\mathbf{j}_{1,1}, \mathbf{j}_{1,2}\right)=\mathbf{j}_{1}}} v^{\frac{1}{2}\left(\left|\mathbf{j}_{1,1}\right|,\left|\mathbf{j}_{1,2}\right|\right)}\left(\mathbf{j}_{1,1} \otimes \mathbf{j}_{1,2}\right) \otimes \mathbf{j}_{2} \\
& =\sum_{\mathbf{j}_{1,1}, \mathbf{j}_{1,2}, \mathbf{j}_{2} \in \mathbf{W}:\left(\mathbf{j}_{1,1}, \mathbf{j}_{1,2}, \mathbf{j}_{2}\right)=\mathbf{h}} v^{\frac{1}{2}\left(\left|\mathbf{j}_{1,1}\right|+\left|\mathbf{j}_{1,2}\right|,\left|\mathbf{j}_{2}\right|\right)} v^{\frac{1}{2}\left(\left|\mathbf{j}_{1,1}\right|,\left|\mathbf{j}_{1,2}\right|\right)} \mathbf{j}_{1,1} \otimes \mathbf{j}_{1,2} \otimes \mathbf{j}_{2}, \\
& (\operatorname{Id} \otimes \Delta) \Delta(\mathbf{h})=\sum_{\mathbf{j}_{1}, \mathbf{j}_{2} \in \mathbf{W}:\left(\mathbf{j}_{1}, \mathbf{j}_{2}\right)=\mathbf{h}} v^{\frac{1}{2}\left(\left|\mathbf{j}_{1}\right|,\left|\mathbf{j}_{2}\right|\right)} \mathbf{j}_{1} \otimes \Delta\left(\mathbf{j}_{2}\right) \\
& =\sum_{\substack{\mathbf{j}_{1}, \mathbf{j}_{2} \in \mathbf{W}: \\
\left(\mathbf{j}_{1}, \mathbf{j}_{2}\right)=\mathbf{h}}} v^{\frac{1}{2}\left(\left|\mathbf{j}_{1}\right|,\left|\mathbf{j}_{2}\right|\right)} \sum_{\substack{\mathbf{j}_{2,1}, \mathbf{j}_{2,2} \in \mathbf{W}: \\
\left(\mathbf{j}_{2,1}, \mathbf{j}_{2,2}\right)=\mathbf{j}_{2}}} v^{\frac{1}{2}\left(\left|\mathbf{j}_{2,1}\right|,\left|\mathbf{j}_{2,2}\right|\right)} \mathbf{j}_{1} \otimes\left(\mathbf{j}_{2,1} \otimes \mathbf{j}_{2,2}\right) \\
& =\sum_{\mathbf{j}_{1}, \mathbf{j}_{2,1}, \mathbf{j}_{2,2}, \in \mathbf{W}:\left(\mathbf{j}_{1}, \mathbf{j}_{2,1}, \mathbf{j}_{2,2}\right)=\mathbf{h}} v^{\frac{1}{2}\left(\left|\mathbf{j}_{1}\right|,\left|\mathbf{j}_{2,1}\right|+\left|\mathbf{j}_{2,2}\right|\right)} v^{\frac{1}{2}\left(\left|\mathbf{j}_{2,1}\right|,\left|\mathbf{j}_{2,2}\right|\right)} \mathbf{j}_{1} \otimes \mathbf{j}_{2,1} \otimes \mathbf{j}_{2,2}
\end{aligned}
$$

But both of these are equal to

$$
\sum_{\mathbf{j}_{1}, \mathbf{j}_{2}, \mathbf{j}_{3}, \in \mathbf{W}:\left(\mathbf{j}_{1}, \mathbf{j}_{2}, \mathbf{j}_{3}\right)=\mathbf{h}} v^{\frac{1}{2}\left(\left|\mathbf{j}_{1}\right|,\left|\mathbf{j}_{2}\right|\right)} v^{\frac{1}{2}\left(\left|\mathbf{j}_{1}\right|,\left|\mathbf{j}_{3}\right|\right)} v^{\frac{1}{2}\left(\left|\mathbf{j}_{2}\right|,\left|\mathbf{j}_{3}\right|\right)} \mathbf{j}_{1} \otimes \mathbf{j}_{2} \otimes \mathbf{j}_{3},
$$

this establishes coassociativity.

To see the compatibility of the shuffle multiplication on $g^{*}$ and $\Delta$ we consider two words $\mathbf{j}_{1}, \mathbf{j}_{2} \in \mathbf{W}$. On one hand we have

$$
\Delta\left(\mathbf{j}_{1} \circ \mathbf{j}_{2}\right)=\sum_{\sigma \in \Sigma_{r, s}} v^{\zeta(\sigma)} \Delta\left(\mathbf{j}_{\sigma}\right)=\sum_{\sigma \in \Sigma_{r, s}} v^{\zeta(\sigma)} \sum_{\mathbf{j}_{\sigma, 1}, \mathbf{j}_{\sigma, 2} \in \mathbf{W}:\left(\mathbf{j}_{\sigma, 1}, \mathbf{j}_{\sigma, 2}\right)=\mathbf{j}_{\sigma}} v^{\frac{1}{2}\left(\left|\mathbf{j}_{\sigma, 1}\right|,\left|\mathbf{j}_{\sigma, 2}\right|\right)} \mathbf{j}_{\sigma, 1} \otimes \mathbf{j}_{\sigma, 2} .
$$

On the other hand we have

$$
\begin{aligned}
& \Delta\left(\mathbf{j}_{1}\right) \circ \Delta\left(\mathbf{j}_{2}\right)=\left(\sum_{\substack{\mathbf{j}_{1,1}, \mathbf{j}_{1,2} \in \mathbf{W}: \\
\left(\mathbf{j}_{1,1}, \mathbf{j}_{1,2}\right)=\mathbf{j}_{1}}} v^{\frac{1}{2}\left(\left|\mathbf{j}_{1,1}\right|,\left|\mathbf{j}_{1,2}\right|\right)} \mathbf{j}_{1,1} \otimes \mathbf{j}_{1,2}\right) \circ\left(\sum_{\substack{\mathbf{j}_{2,1}, \mathbf{j}_{2,2} \in \mathbf{W}: \\
\left(\mathbf{j}_{2,1}, \mathbf{j}_{2,2}\right)=\mathbf{j}_{2}}} v^{\frac{1}{2}\left(\left|\mathbf{j}_{2,1}\right|,\left|\mathbf{j}_{2,2}\right|\right)} \mathbf{j}_{2,1} \otimes \mathbf{j}_{2,2}\right)
\end{aligned}
$$

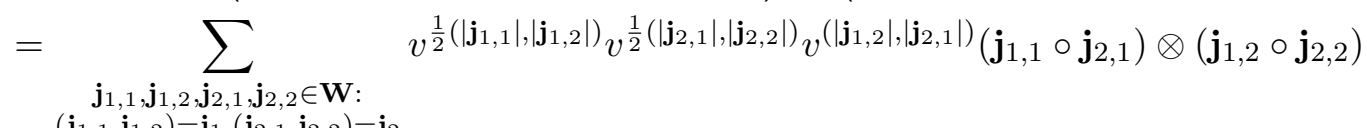

$$
\begin{aligned}
& \left(\mathbf{j}_{1,1}, \mathbf{j}_{1,2}\right)=\mathbf{j}_{1},\left(\mathbf{j}_{2,1}, \mathbf{j}_{2,2}\right)=\mathbf{j}_{2} \\
& =\sum_{\substack{\left.\mathbf{j}_{1,1}, \mathbf{j}_{1,2}, \mathbf{j}_{2,1}, \mathbf{j}_{2,2} \in \mathbf{W}: \\
\left(\mathbf{j}_{1,1}, \mathbf{j}_{1,2}\right)=\mathbf{j}_{1}, \mathbf{j}_{2}, \mathbf{j}_{2,2}\right)=\mathbf{j}_{2}}} \sum_{\sigma_{1} \in \Sigma_{r_{1}, s_{1}}} \sum_{\sigma_{2} \in \Sigma_{r_{2}, s_{2}}} v^{\frac{1}{2}\left(\left|\mathbf{j}_{1,1}\right|,\left|\mathbf{j}_{1,2}\right|\right)} v^{\frac{1}{2}\left(\left|\mathbf{j}_{2,1}\right|,\left|\mathbf{j}_{2,2}\right|\right)} v^{\left(\left|\mathbf{j}_{1,2}\right|,\left|\mathbf{j}_{2,1}\right|\right)} \\
& \left(\mathbf{j}_{1,1}, \mathbf{j}_{1,2}\right)=\mathbf{j}_{1},\left(\mathbf{j}_{2,1}, \mathbf{j}_{2,2}\right)=\mathbf{j}_{2} \\
& \times v^{\zeta\left(\sigma_{1}\right)} v^{\zeta\left(\sigma_{2}\right)} \mathbf{j}_{\sigma_{1}, 1} \otimes \mathbf{j}_{\sigma_{2}, 2},
\end{aligned}
$$

where we wrote $r_{i}$ and $s_{i}(i=1,2)$ for the lengths of $\mathbf{j}_{i, 1}$ and $\mathbf{j}_{i, 2}$ respectively. There is an obvious bijection between pairs consisting of a shuffle $\sigma \in \Sigma_{r, s}$ together with a partition $\left(\mathbf{j}_{\sigma, 1}, \mathbf{j}_{\sigma, 2}\right)$ of $\mathbf{j}_{\sigma}$ and quadruples consisting of partitions of $\mathbf{j}_{1}$ and $\mathbf{j}_{2}$ together with shuffles $\sigma_{1} \in \Sigma_{r_{1}, s_{1}}$ and $\sigma_{2} \in \Sigma_{r_{2}, s_{2}}$. Indeed, reading off the contributions in $\mathbf{j}_{\sigma, 1}$ and $\mathbf{j}_{\sigma, 2}$ coming from $\mathbf{j}_{1}$ and $\mathbf{j}_{2}$ produce the desired partitions while the relative positions of these contributions produce the desired shuffles in $\Sigma_{r_{1}, s_{1}}$ and $\Sigma_{r_{2}, s_{2}}$.

Notice that under this bijection we have

$$
\zeta(\sigma)=\zeta\left(\sigma_{1}\right)+\zeta\left(\sigma_{2}\right)+\frac{1}{2}\left(\left|\mathbf{j}_{1,2}\right|,\left|\mathbf{j}_{2,1}\right|\right)-\frac{1}{2}\left(\left|\mathbf{j}_{1,1}\right|,\left|\mathbf{j}_{2,2}\right|\right)
$$

and

$$
\left(\left|\mathbf{j}_{\sigma, 1}\right|,\left|\mathbf{j}_{\sigma, 2}\right|\right)=\left(\left|\mathbf{j}_{1,1}\right|,\left|\mathbf{j}_{1,2}\right|\right)+\left(\left|\mathbf{j}_{1,1}\right|,\left|\mathbf{j}_{2,2}\right|\right)+\left(\left|\mathbf{j}_{2,1}\right|,\left|\mathbf{j}_{1,2}\right|\right)+\left(\left|\mathbf{j}_{2,1}\right|,\left|\mathbf{j}_{2,2}\right|\right) .
$$

An easy inspection now shows that $\Delta\left(\mathbf{j}_{1} \circ \mathbf{j}_{2}\right)=\Delta\left(\mathbf{j}_{1}\right) \circ \Delta\left(\mathbf{j}_{2}\right)$ as claimed. 
It will be useful to understand iterated shuffle multiplication of length one words.

Lemma 6.4. For any word $\mathbf{j} \in \mathbf{W}$ we have

$$
\left(j_{1}\right) \circ \cdots \circ\left(j_{r}\right)=\sum_{\sigma \in \Sigma_{r}} v^{\eta(\sigma)} \mathbf{j}_{\sigma}
$$

where $\eta(\sigma)=\frac{1}{2} \sum_{\substack{k<\ell \\ \sigma_{\ell}^{-1}<\sigma_{k}^{-1}}}\left(\alpha_{j_{k}}, \alpha_{j_{\ell}}\right)-\frac{1}{2} \sum_{\substack{k<\ell \\ \sigma_{k}^{-1}<\sigma_{\ell}^{-1}}}\left(\alpha_{j_{k}}, \alpha_{j_{\ell}}\right)$.

Proof. We work by induction on $r$ beginning with the case $r=2$ :

$$
\left(j_{1}\right) \circ\left(j_{2}\right)=v^{-\frac{1}{2}\left(\alpha_{j_{1}}, \alpha_{j_{2}}\right)}\left(j_{1}, j_{2}\right)+v^{\frac{1}{2}\left(\alpha_{j_{1}}, \alpha_{j_{2}}\right)}\left(j_{2}, j_{1}\right)
$$

which is easily seen to verify the claim. For $r>2$, using the inductive hypothesis for $r$ we have

$$
\left(j_{1}\right) \circ \cdots \circ\left(j_{r+1}\right)=\sum_{\sigma \in \Sigma_{r}} v^{\eta(\sigma)} \mathbf{j}_{\sigma} \circ\left(j_{r+1}\right)=\sum_{\substack{\sigma \in \Sigma_{r} \\ \tau \in \Sigma_{r, 1}}} v^{\eta(\sigma)+\zeta(\tau)} \mathbf{j}_{\tilde{\sigma} \cdot \tau}=\sum_{\sigma \in \Sigma_{r+1}} v^{\eta(\sigma)} \mathbf{j}_{\sigma} .
$$

Lemma 6.5. Taking $j_{\ell}=i$ for all $\ell$ in the definition of $\eta(\sigma)$ we have $\sum_{\sigma \in \Sigma_{r}} v^{\eta(\sigma)}=[r]_{i}^{!}$.

Proof. For $r=1$ there is nothing to show, so we begin our induction with $r=2$ :

$$
\sum_{\sigma \in \Sigma_{2}} v^{\eta(\sigma)}=v^{d_{i}}+v^{-d_{i}}=[2]_{i} .
$$

Now assume the result holds for $r$, then we complete the induction by a direct calculation:

$$
\begin{aligned}
\sum_{\sigma \in \Sigma_{r+1}} v^{\eta(\sigma)} & =\sum_{1 \leq k \leq r+1} \sum_{\sigma \in \Sigma_{r+1}, \sigma_{k}^{-1}=r+1} v^{\eta(\sigma)}=\sum_{1 \leq k \leq r+1} v^{(r+2-2 k) d_{i}} \sum_{\sigma \in \Sigma_{r}} v^{\eta(\sigma)} \\
& =[r+1]_{i}[r]_{i}^{!}
\end{aligned}
$$

We then get the following immediate consequence.

Corollary 6.6. For any $r$ we have $(i)^{\circ[r]}=\left(i^{r}\right)$ where $(i)^{\circ[r]}=(i)^{\circ r} /[r]_{i}^{!}$.

It will also be useful to understand the shuffle of two isotypic words, the main ingredient is the following.

Lemma 6.7. Taking $j_{\ell}=i$ for all $\ell$ in the definition of $\zeta(\sigma)$, we have

$$
\sum_{\sigma \in \Sigma_{r, s}} v^{\zeta(\sigma)}=\left[\begin{array}{c}
r+s \\
r
\end{array}\right]_{i}
$$

for any nonnegative integers $r$ and $s$.

Proof. We proceed by induction, the base of the induction $r=s=0$ being clear. The induction step is given by the following use of the second quantum Pascal identity from Lemma 2.1(2a):

$$
\begin{aligned}
\sum_{\sigma \in \Sigma_{r, s}} v^{\zeta(\sigma)} & =\sum_{\sigma \in \Sigma_{r, s}, \sigma_{r+s}=r} v^{\zeta(\sigma)}+\sum_{\sigma \in \Sigma_{r, s}, \sigma_{r+s}=r+s} v^{\zeta(\sigma)}=v_{i}^{s} \sum_{\sigma \in \Sigma_{r-1, s}} v^{\zeta(\sigma)}+v_{i}^{-r} \sum_{\sigma \in \Sigma_{r, s-1}} v^{\zeta(\sigma)} \\
& =v_{i}^{s}\left[\begin{array}{c}
r+s-1 \\
r-1
\end{array}\right]_{i}+v_{i}^{-r}\left[\begin{array}{c}
r+s-1 \\
r
\end{array}\right]_{i}=\left[\begin{array}{c}
r+s \\
r
\end{array}\right]_{i} .
\end{aligned}
$$


This allows us to prove the following pair of results which are essential for obtaining the embedding of the quantum group $\mathcal{A}_{v}[N]$ in the quantum shuffle algebra $g^{*}$.

Lemma 6.8. For $0 \leq r \leq n$ we have

$$
\begin{aligned}
(i)^{\circ}[r] & \circ(j) \circ(i)^{\circ[n-r]} \\
& =\sum_{s=0}^{r} \sum_{t=0}^{n-r} v_{i}^{-s\left(a_{i j}+n\right)+t a_{i j}-\frac{1}{2} n a_{i j}}\left[\begin{array}{c}
s+t \\
s
\end{array}\right]_{i}\left[\begin{array}{c}
n-s-t \\
r-s
\end{array}\right]_{i} v_{i}^{r\left(a_{i j}+s+t\right)}\left(i^{s+t}, j, i^{n-s-t}\right) .
\end{aligned}
$$

Proof. By Corollary 6.6 we have $(i)^{\circ[r]} \circ(j) \circ(i)^{\circ[n-r]}=\left(i^{r}\right) \circ(j) \circ\left(i^{n-r}\right)$. In the product $\left(i^{r}\right) \circ(j) \circ\left(i^{n-r}\right)$ we denote by $r-s$ the number of the first $r i$ 's that shuffle to the right of $j$ and denote by $t$ the number of the last $n-r i$ 's that shuffle to the left of $j$. The coefficient of $\left(i^{s}, j, i^{r-s}\right)$ in the product $\left(i^{r}\right)(j)$ is $v_{i}^{\frac{1}{2}(r-s) a_{i j}-\frac{1}{2} s a_{i j}}$. Using Lemma 6.7, the coefficient of $\left(i^{s+t}, j, i^{n-s-t}\right)$ in the product $\left(i^{s}, j, i^{r-s}\right)\left(i^{n-r}\right)$ is

$$
v_{i}^{(r-s) t-s(n-r-t)}\left[\begin{array}{c}
s+t \\
s
\end{array}\right]_{i}\left[\begin{array}{c}
n-s-t \\
r-s
\end{array}\right]_{i} v_{i}^{\frac{1}{2} t a_{i j}-\frac{1}{2}(n-r-t) a_{i j}} .
$$

Thus, after simplifying slightly, the coefficient of $\left(i^{s+t}, j, i^{n-s-t}\right)$ in the product $\left(i^{r}\right) \circ(j) \circ\left(i^{n-r}\right)$ becomes

$$
v_{i}^{-s\left(a_{i j}+n\right)+t a_{i j}-\frac{1}{2} n a_{i j}}\left[\begin{array}{c}
s+t \\
s
\end{array}\right]_{i}\left[\begin{array}{c}
n-s-t \\
r-s
\end{array}\right]_{i} v_{i}^{r\left(a_{i j}+s+t\right)}
$$

as claimed

Corollary 6.9. For any $i \neq j \in I$ the following quantum Serre relation holds in $g^{*}$ :

$$
\sum_{r=0}^{1-a_{i j}}(-1)^{r}(i)^{\circ[r]} \circ(j) \circ(i)^{\circ\left[1-a_{i j}-r\right]}=0 .
$$

Proof. Using Lemma 6.8 with $n=1-a_{i j}$ we may expand the left hand side of (6.2) as follows. We abbreviate $\mathbf{i}_{s, t}=\left(i^{s+t}, j, i^{1-a_{i j}-s-t}\right)$ and in each equality we simply change the order of summation:

$$
\begin{aligned}
& \sum_{r=0}^{1-a_{i j}}(-1)^{r}(i)^{\circ[r]} \circ(j) \circ(i)^{\circ\left[1-a_{i j}-r\right]} \\
& =\sum_{r=0}^{1-a_{i j}} \sum_{s=0}^{r} \sum_{t=0}^{1-a_{i j}-r}(-1)^{r} v_{i}^{-s+t a_{i j}-\frac{1}{2}\left(1-a_{i j}\right) a_{i j}}\left[\begin{array}{c}
s+t \\
s
\end{array}\right]_{i}\left[\begin{array}{c}
1-a_{i j}-s-t \\
r-s
\end{array}\right]_{i} v_{i}^{r\left(a_{i j}+s+t\right)} \mathbf{i}_{s, t} \\
& =\sum_{s=0}^{1-a_{i j}} \sum_{r=s}^{1-a_{i j}} \sum_{t=0}^{1-a_{i j}-r}(-1)^{r} v_{i}^{-s+t a_{i j}-\frac{1}{2}\left(1-a_{i j}\right) a_{i j}}\left[\begin{array}{c}
s+t \\
s
\end{array}\right]_{i}\left[\begin{array}{c}
1-a_{i j}-s-t \\
r-s
\end{array}\right]_{i} v_{i}^{r\left(a_{i j}+s+t\right)} \mathbf{i}_{s, t} \\
& =\sum_{s=0}^{1-a_{i j}} \sum_{r=0}^{1-a_{i j}-s} \sum_{t=0}^{r}(-1)^{1-a_{i j}-r} v_{i}^{-s+t a_{i j}-\frac{1}{2}\left(1-a_{i j}\right) a_{i j}}\left[\begin{array}{c}
s+t \\
s
\end{array}\right]_{i}\left[\begin{array}{c}
1-a_{i j}-s-t \\
1-a_{i j}-r-s
\end{array}\right]_{i} \\
& \times v_{i}^{\left(1-a_{i j}-r\right)\left(a_{i j}+s+t\right)} \mathbf{i}_{s, t} \\
& =\sum_{s=0}^{1-a_{i j}} \sum_{t=0}^{1-a_{i j}-s} \sum_{r=t}^{1-a_{i j}-s}(-1)^{1-a_{i j}-r} v_{i}^{-s+t a_{i j}-\frac{1}{2}\left(1-a_{i j}\right) a_{i j}}\left[\begin{array}{c}
s+t \\
s
\end{array}\right]_{i}\left[\begin{array}{c}
1-a_{i j}-s-t \\
1-a_{i j}-r-s
\end{array}\right]_{i} \\
& \times v_{i}^{\left(1-a_{i j}-r\right)\left(a_{i j}+s+t\right)} \mathbf{i}_{s, t}
\end{aligned}
$$




$$
\begin{aligned}
= & \sum_{s=0}^{1-a_{i j}} \sum_{t=0}^{1-a_{i j}-s} \sum_{r=0}^{1-a_{i j}-s-t}(-1)^{r+s} v_{i}^{-s+t a_{i j}-\frac{1}{2}\left(1-a_{i j}\right) a_{i j}}\left[\begin{array}{c}
s+t \\
s
\end{array}\right]_{i}\left[\begin{array}{c}
1-a_{i j}-s-t \\
r
\end{array}\right]_{i} \\
& \times v_{i}^{(r+s)\left(a_{i j}+s+t\right)} \mathbf{i}_{s, t} \\
= & \sum_{s=0}^{1-a_{i j}} \sum_{t=0}^{1-a_{i j}-s}(-1)^{s} v_{i}^{-s\left(1-a_{i j}-s-t\right)+t a_{i j}-\frac{1}{2}\left(1-a_{i j}\right) a_{i j}}\left[\begin{array}{c}
s+t \\
s
\end{array}\right]_{i} \mathbf{i}_{s, t} \\
& \times \sum_{r=0}^{1-a_{i j}-s-t}(-1)^{r} v_{i}^{r-r\left(1-a_{i j}-s-t\right)}\left[\begin{array}{c}
1-a_{i j}-s-t \\
r
\end{array}\right]_{i} .
\end{aligned}
$$

By Lemma 2.1, the last inner summation is zero unless $s+t=1-a_{i j}$, thus the above becomes:

$$
\begin{aligned}
& \sum_{s=0}^{1-a_{i j}}(-1)^{s} v_{i}^{\frac{1}{2}\left(1-a_{i j}\right) a_{i j}-s a_{i j}}\left[\begin{array}{c}
1-a_{i j} \\
s
\end{array}\right]_{i} \mathbf{i}_{s, 1-a_{i j}-s} \\
& \quad=v_{i}^{\frac{1}{2}\left(1-a_{i j}\right) a_{i j}}\left(i^{1-a_{i j}}, j\right) \sum_{s=0}^{1-a_{i j}}(-1)^{s} v_{i}^{s\left(1-a_{i j}\right)-s}\left[\begin{array}{c}
1-a_{i j} \\
s
\end{array}\right]_{i}
\end{aligned}
$$

which is zero by Lemma 2.1 .

The following theorem can be proven from Corollary 6.9 by the same argument as in the proof of [23, Theorem 4].

Theorem 6.10. There exists an injective algebra homomorphism $\kappa: \mathcal{A}_{v}[N] \hookrightarrow g^{*}$ defined on generators by $\kappa\left(x_{i}\right)=(i)$.

We now introduce certain derivations acting on $g^{*}$. For $i \in I$ define maps $\theta_{i}, \theta_{i}^{*}: g^{*} \rightarrow g^{*}$ by linearly extending the following:

$$
\begin{aligned}
& \theta_{i}(\mathbf{j})= \begin{cases}v^{\frac{1}{2}\left(|\mathbf{j}|-\alpha_{i}, \alpha_{i}\right)}\left(j_{1}, \ldots, j_{r-1}\right) & \text { if } j_{r}=i, \\
0 & \text { otherwise, }\end{cases} \\
& \theta_{i}^{*}(\mathbf{j})= \begin{cases}v^{\frac{1}{2}\left(\alpha_{i},|\mathbf{j}|-\alpha_{i}\right)}\left(j_{2}, \ldots, j_{r}\right) & \text { if } j_{1}=i, \\
0 & \text { otherwise }\end{cases}
\end{aligned}
$$

\section{Lemma 6.11.}

(a) For any $i \in I$ the map $\theta_{i}: g^{*} \rightarrow g^{*}$ is a twisted derivation with respect to the shuffle product, i.e. $\theta_{i}$ satisfies

$$
\theta_{i}\left(\mathbf{j}_{1} \circ \mathbf{j}_{2}\right)=v^{\left(\alpha_{i},\left|\mathbf{j}_{2}\right|\right)} \theta_{i}\left(\mathbf{j}_{1}\right) \circ \mathbf{j}_{2}+\mathbf{j}_{1} \circ \theta_{i}\left(\mathbf{j}_{2}\right) \quad \text { for any } \mathbf{j}_{1}, \mathbf{j}_{2} \in \mathbf{W}
$$

(b) For any $i \in I$ the map $\theta_{i}^{*}: g^{*} \rightarrow g^{*}$ is a twisted derivation with respect to the shuffle product, i.e. $\theta_{i}^{*}$ satisfies

$$
\theta_{i}^{*}\left(\mathbf{j}_{1} \circ \mathbf{j}_{2}\right)=\theta_{i}^{*}\left(\mathbf{j}_{1}\right) \circ \mathbf{j}_{2}+v^{\left(\left|\mathbf{j}_{1}\right|, \alpha_{i}\right)} \mathbf{j}_{1} \circ \theta_{i}^{*}\left(\mathbf{j}_{2}\right) \quad \text { for any } \quad \mathbf{j}_{1}, \mathbf{j}_{2} \in \mathbf{W} .
$$

Proof. The results follow from a direct calculation:

$$
\theta_{i}\left(\mathbf{j}_{1} \circ \mathbf{j}_{2}\right)=\sum_{\sigma \in \Sigma_{r, s}} v^{\zeta(\sigma)} \theta_{i}\left(\mathbf{j}_{\sigma}\right)=\sum_{\substack{\sigma \in \Sigma_{r, s} \\ \sigma_{r}^{-1}=r+s}} v^{\zeta(\sigma)} \theta_{i}\left(\mathbf{j}_{\sigma}\right)+\sum_{\substack{\sigma \in \Sigma_{r, s} \\ \sigma_{r+s}^{-1}=r+s}} v^{\zeta(\sigma)} \theta_{i}\left(\mathbf{j}_{\sigma}\right)
$$




$$
=v^{\left(\alpha_{j_{r}}, \mathbf{j}_{2} \mid\right)} \theta_{i}\left(\mathbf{j}_{1}\right) \circ \mathbf{j}_{2}+\mathbf{j}_{1} \circ \theta_{i}\left(\mathbf{j}_{2}\right)
$$

and similarly:

$$
\begin{aligned}
\theta_{i}^{*}\left(\mathbf{j}_{1} \circ \mathbf{j}_{2}\right) & =\sum_{\sigma \in \Sigma_{r, s}} v^{\zeta(\sigma)} \theta_{i}^{*}\left(\mathbf{j}_{\sigma}\right)=\sum_{\substack{\sigma \in \Sigma_{r, s} \\
\sigma_{1}^{-1}=1}} v^{\zeta(\sigma)} \theta_{i}^{*}\left(\mathbf{j}_{\sigma}\right)+\sum_{\substack{\sigma \in \Sigma_{r, s} \\
\sigma_{r+1}^{-1}=1}} v^{\zeta(\sigma)} \theta_{i}^{*}\left(\mathbf{j}_{\sigma}\right) \\
& =\theta_{i}^{*}\left(\mathbf{j}_{1}\right) \circ \mathbf{j}_{2}+v^{\left(\left|\mathbf{j}_{1}\right|, \alpha_{j_{r+1}}\right)} \mathbf{j}_{1} \circ \theta_{i}^{*}\left(\mathbf{j}_{2}\right) .
\end{aligned}
$$

\subsection{Generalized Feigin homomorphisms}

Given that we have extended the Feigin homomorphisms to the dual Hall-Ringel algebra, one well-known container for the quantum group, it is natural to try to establish extensions of the Feigin homomorphisms to the quantum shuffle algebra. Our proposed extension $\bar{\Psi}_{\mathbf{i}}: g^{*} \rightarrow P_{\mathbf{i}}$ agrees with the classical Feigin homomorphisms on the generators $(i)(i \in I)$ of $g^{*}$, thus we suspect that it will play the same simplifying role as the classical Feigin homomorphisms and our generalized Feigin homomorphisms from Section 5.1.

Fix a word $\mathbf{i}=\left(i_{1}, \ldots, i_{m}\right) \in I^{m}$. We define $\bar{\Psi}_{\mathbf{i}}: g^{*} \rightarrow P_{\mathbf{i}}$ by extending linearly the following map:

$$
\bar{\Psi}_{\mathbf{i}}(\mathbf{j})=\sum_{\substack{\mathbf{a} \in \mathbb{Z}_{\geq 0}^{m} \\\left(i_{1}^{a_{1}}, \ldots, i_{m}^{a_{m}}\right)=\mathbf{j}}} \frac{1}{\left[a_{1}\right]_{i_{1}}^{!} \cdots\left[a_{m}\right]_{i_{m}}^{!}} t^{\mathbf{a}} .
$$

Notice that $\bar{\Psi}_{\mathbf{i}}(\mathbf{j})$ is a bar-invariant element of $P_{\mathbf{i}}$, hence the choice of notation. The following result asserts that this map defines a homomorphism of algebras.

Theorem 6.12. The map $\bar{\Psi}_{\mathbf{i}}$ is an algebra homomorphism from $g^{*}$ to $P_{\mathbf{i}}$.

Proof. We begin with the isotypic case $j_{k}=i(1 \leq k \leq r+s)$ and $i_{\ell}=i(1 \leq \ell \leq m)$. In this case the identity $\bar{\Psi}_{\mathbf{i}}\left(\mathbf{j}_{1} \circ \mathbf{j}_{2}\right)=\bar{\Psi}_{\mathbf{i}}\left(\mathbf{j}_{1}\right) \bar{\Psi}_{\mathbf{i}}\left(\mathbf{j}_{2}\right)$ reduces to the following:

$$
\sum_{\sigma \in \Sigma_{r, s}} v^{\zeta(\sigma)}=\sum_{\substack{\mathbf{a}, \mathbf{a}^{\prime} \in \mathbb{Z}_{\geq 0}^{m} \\
a_{1}+\cdots+a_{m}^{\prime}=r \\
a_{1}^{\prime}+\cdots+a_{m}^{\prime}=s}} v_{i}^{\sum_{k<\ell} a_{k}^{\prime} a_{\ell}-\sum_{k<\ell} a_{k} a_{\ell}^{\prime}}\left[\begin{array}{c}
c_{1} \\
a_{1}
\end{array}\right]_{i} \ldots\left[\begin{array}{c}
c_{m} \\
a_{m}
\end{array}\right]_{i}
$$

for any $\mathbf{c} \in \mathbb{Z}_{>0}^{m}$ with $c_{1}+\cdots+c_{m}=r+s$. This in turn easily follows from Lemma 6.7 by repeated application of the bar-invariant subspace identity in Lemma 2.1.

Moving on to the general case we must establish the identity:

$$
\sum_{\substack{\sigma \in \sum_{r, s} \\
\left(i_{1}^{c_{1}}, \ldots, i_{m}^{c m}\right)=\mathbf{j}_{\sigma}}} v^{\zeta(\sigma)}=\sum_{\substack{\mathbf{a}, \mathbf{a}^{\prime} \in \mathbb{Z}_{\geq 0}^{m}, \mathbf{a}+\mathbf{a}^{\prime}=\mathbf{c} \\
\left(i_{1}^{a_{1}}, \ldots, i_{m}^{a}\right)=\mathbf{j}_{1} \\
\left(i_{1}^{\prime}, \ldots, a_{m}^{\prime}\right)=\mathbf{j}_{2}}} v^{\frac{1}{2} \sum_{k<\ell} a_{k}^{\prime} a_{\ell}\left(\alpha_{i_{k}}, \alpha_{i_{\ell}}\right)-\frac{1}{2} \sum_{\ell<k} a_{\ell} a_{k}^{\prime}\left(\alpha_{i_{k}}, \alpha_{i_{\ell}}\right)}\left[\begin{array}{c}
c_{1} \\
a_{1}
\end{array}\right]_{i_{1}} \ldots\left[\begin{array}{c}
c_{m} \\
a_{m}
\end{array}\right]_{i_{m}}
$$

for any $\mathbf{c} \in \mathbb{Z}_{\geq 0}^{m}$.

For $\mathbf{a}+\mathbf{a}^{\prime}=\mathbf{c}$ the term $\left(i_{1}^{c_{1}}, \ldots, i_{m}^{c_{m}}\right)$ in the shuffle of $\mathbf{j}_{1}=\left(i_{1}^{a_{1}}, \ldots, i_{m}^{a_{m}}\right)$ and $\mathbf{j}_{2}=\left(i_{1}^{a_{1}^{\prime}}, \ldots, i_{m}^{a_{m}^{\prime}}\right)$ is obtained by two steps:

- shuffling the subword $i_{k}^{a_{k}^{\prime}}$ of $\mathbf{j}_{2}$ past each subword $i_{\ell}^{a_{\ell}}(\ell>k)$ of $\mathbf{j}_{1}$;

- shuffling the subword $i_{k}^{a_{k}^{\prime}}$ of $\mathbf{j}_{2}$ into the subword $i_{k}^{a_{k}}$ of $\mathbf{j}_{1}$. 
The first shuffle process produces the exponent $\frac{1}{2} \sum_{k<\ell} a_{k}^{\prime} a_{\ell}\left(\alpha_{i_{k}}, \alpha_{i_{\ell}}\right)$ while the exponent $-\frac{1}{2} \sum_{\ell<k} a_{\ell} a_{k}^{\prime}\left(\alpha_{i_{k}}, \alpha_{i_{\ell}}\right)$ is a consequence of the subword $i_{k}^{a_{k}^{\prime}}$ of $\mathbf{j}_{2}$ not shuffling past any subword $i_{\ell}^{a_{\ell}}(\ell<k)$ of $\mathbf{j}_{1}$. The second shuffle process above produces the factor $\left[\begin{array}{c}c_{k} \\ a_{k}\end{array}\right]_{i_{k}}$ by the isotypic case. Comparing with the right hand side of the desired equality completes the proof.

\section{Quantum shuffle characters}

The results of Sections 3, 6.1, and 5.1 have established the commutativity of the front two faces in the tetrahedron below:

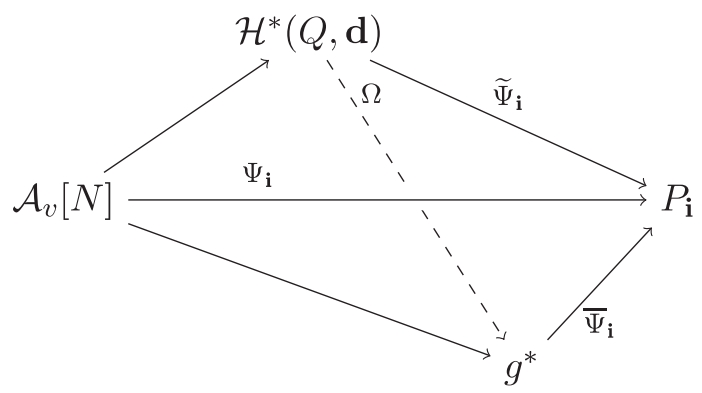

Our goal of this section is to complete this diagram, that is define the quantum shuffle character homomorphism $\Omega: \mathcal{H}^{*}(Q, \mathbf{d}) \rightarrow g^{*}$ making the remaining two faces of the tetrahedron commute.

For a word $\mathbf{j}=\left(j_{1}, \ldots, j_{r}\right)$ write $\mathcal{F}_{\mathbf{j}}(V)$ for the set of flags in $V$ of type $\mathbf{j}$ :

$$
\mathcal{F}_{\mathbf{j}}(V)=\left\{0=V_{r} \subset V_{r-1} \subset \cdots \subset V_{1} \subset V_{0}=V: V_{k-1} / V_{k} \cong S_{j_{k}} \text { for } 1 \leq k \leq r\right\} .
$$

Then for a basis vector $[V]^{*} \in \mathcal{H}^{*}(Q, \mathbf{d})$ we define $\Omega\left([V]^{*}\right) \in g^{*}$ as a certain generating function for counting flags in $V$ :

$$
\Omega\left([V]^{*}\right)=\sum_{\mathbf{j} \in \mathbf{W}} v^{-\sum_{k<\ell}\left\langle\alpha_{j_{\ell}}, \alpha_{j_{k}}\right\rangle}\left|\mathcal{F}_{\mathbf{j}}(V)\right| \cdot \mathbf{j} .
$$

This map $\Omega: \mathcal{H}^{*}(Q, \mathbf{d}) \rightarrow g^{*}$ turns out to preserve a fair amount of structure.

Theorem 7.1. The map $\Omega: \mathcal{H}^{*}(Q, \mathbf{d}) \rightarrow g^{*}$ given by (7.2) is a homomorphism of twisted bialgebras.

Remark 7.2. In general, $\Omega$ will not be injective. For example, every member of the $\mathbb{P}^{1}$-family of indecomposable representations with dimension vector $(1,1)$ for the 2-Kronecker quiver will have the same image under $\Omega$.

Proof. We begin by showing that $\Omega$ is an algebra homomorphism. Recall the rescaling

$$
[V]^{*}=v^{-\frac{1}{2}\langle|V|,|V|\rangle+\frac{1}{2} \sum_{i=1}^{n} d_{i} v_{i}} \delta_{[V]}
$$

The product $[U]^{*}[W]^{*}$ can then be computed in this same basis as

$$
\begin{aligned}
{[U]^{*}[W]^{*} } & =v^{-\frac{1}{2}\langle|U|+|W|,|U|+|W|\rangle+\frac{1}{2}\langle|U|,|W|\rangle+\frac{1}{2}\langle|W|,|U|\rangle+\frac{1}{2} \sum_{i=1}^{n} d_{i}\left(u_{i}+w_{i}\right)} \delta_{[U]} \delta_{[W]} \\
& =\sum_{[V]} v^{-\frac{1}{2}\langle|V|,|V|\rangle+\frac{1}{2}\langle|U|,|W|\rangle+\frac{1}{2}\langle|W|,|U|\rangle+\frac{1}{2} \sum_{i=1}^{n} d_{i} v_{i}} v^{\langle|U|,|W|\rangle}
\end{aligned}
$$




$$
\begin{aligned}
& \times \frac{|\operatorname{Aut}(U)||\operatorname{Aut}(W)|}{|\operatorname{Aut}(V)|} \cdot\left|\mathcal{F}_{U W}^{V}\right| \cdot \delta_{[V]} \\
= & \sum_{[V]} v^{\frac{3}{2}\langle|U|,|W|\rangle+\frac{1}{2}\langle|W|,|U|\rangle} \frac{|\operatorname{Aut}(U)||\operatorname{Aut}(W)|}{|\operatorname{Aut}(V)|} \cdot\left|\mathcal{F}_{U W}^{V}\right| \cdot[V]^{*}
\end{aligned}
$$

Thus the equality $\Omega\left([U]^{*}[W]^{*}\right)=\Omega\left([U]^{*}\right) \Omega\left([W]^{*}\right)$ is equivalent to the following interesting collection of identities involving flags.

Lemma 7.3. For every word $\mathbf{h} \in \mathbf{W}$ we have

$$
\begin{aligned}
& \sum_{[V]} v^{\frac{3}{2}\langle|U|,|W|\rangle+\frac{1}{2}\langle|W|,|U|\rangle-\sum_{k<\ell}\left\langle\alpha_{h_{\ell}}, \alpha_{h_{k}}\right\rangle} \frac{|\operatorname{Aut}(U)||\operatorname{Aut}(W)|}{|\operatorname{Aut}(V)|}\left|\mathcal{F}_{U W}^{V}\right|\left|\mathcal{F}_{\mathbf{h}}(V)\right| \\
& \quad=\sum_{\substack{\mathbf{j}_{1}, \mathbf{j}_{2} \in \mathbf{W} \\
\sigma \in \Sigma_{\mathbf{h t}(U), \operatorname{ht}(W)}: \mathbf{j}_{\sigma}=\mathbf{h}}} v^{-\sum_{1 \leq k<\ell \leq t}\left\langle\alpha_{j_{\ell},}, \alpha_{j_{k}}\right\rangle-\sum_{t+1 \leq k<\ell \leq r+s}\left\langle\alpha_{j_{\ell}}, \alpha_{j_{k}}\right\rangle+\zeta(\sigma)}\left|\mathcal{F}_{\mathbf{j}_{1}}(U)\right|\left|\mathcal{F}_{\mathbf{j}_{2}}(W)\right|,
\end{aligned}
$$

where we abbreviated $t=\mathrm{ht}(U)$.

Proof. This essentially follows from the compatibility of multiplication and comultiplication in the Hall-Ringel algebra $\mathcal{H}(Q, \mathbf{d})$. Using the definitions it is easy to compute the following composition of comultiplication and iterated multiplication:

$$
\begin{aligned}
& \Delta\left(\left[S_{h_{1}}\right] \cdots\left[S_{h_{r+s}}\right]\right)=\sum_{[V]} v^{\sum_{k<\ell}\left\langle\alpha_{h_{k}}, \alpha_{h_{\ell}}\right\rangle}\left|\mathcal{F}_{\mathbf{h}}(V)\right| \Delta([V]) \\
& \quad=\sum_{[U],[V],[W]} v^{\langle|U|,|W|\rangle+\sum_{k<\ell}\left\langle\alpha_{h_{k}}, \alpha_{h_{\ell}}\right\rangle} \frac{|\operatorname{Aut}(U)||\operatorname{Aut}(W)|}{|\operatorname{Aut}(V)|}\left|\mathcal{F}_{U W}^{V}\right|\left|\mathcal{F}_{\mathbf{h}}(V)\right| \cdot[U] \otimes[V] .
\end{aligned}
$$

Theorem 5.4 asserts that this is the same as the following iterated multiplication of comultiplications:

$$
\begin{gathered}
\Delta\left(\left[S_{h_{1}}\right]\right) \cdots \Delta\left(\left[S_{h_{r+s}}\right]\right)=\left(\left[S_{h_{1}}\right] \otimes 1+1 \otimes\left[S_{h_{1}}\right]\right) \cdots\left(\left[S_{h_{r+s}}\right] \otimes 1+1 \otimes\left[S_{h_{r+s}}\right]\right) \\
=\sum_{\substack{[U],[W]: \\
\operatorname{ht}(U)+\operatorname{ht}(W)=r+s \\
\sigma \in \sum_{\mathrm{ht}(U), \mathrm{ht}(W)} \\
\mathbf{j}_{1}, \mathbf{j}_{2} \in \mathbf{W}: \mathbf{j}_{\sigma}=\mathbf{h}}}^{E} v^{E}\left|\mathcal{F}_{\mathbf{j}_{1}}(U)\right|\left|\mathcal{F}_{\mathbf{j}_{2}}(W)\right| \cdot[U] \otimes[W], \\
\end{gathered}
$$

with exponent $E=\sum_{\substack{1 \leq k<\ell \leq t \\ j_{k}}}\left\langle\alpha_{j_{\ell}}\right\rangle+\sum_{t+1 \leq k<\ell \leq r+s}\left\langle\alpha_{j_{k}}, \alpha_{j_{\ell}}\right\rangle+\sum_{\substack{1 \leq k \leq t \\ t+1 \leq \ell \leq r+s \\ \sigma_{k}^{-1}>\sigma_{\ell}^{-1}}}\left(\alpha_{j_{k}}, \alpha_{j_{\ell}}\right)$, where we abbreviate $t=\operatorname{ht}(U)$.

A few remarks seem necessary to clarify the second equality above. To obtain a fixed $[U]$ and $[W]$ we must take from the product

$$
\left(\left[S_{h_{1}}\right] \otimes 1+1 \otimes\left[S_{h_{1}}\right]\right) \cdots\left(\left[S_{h_{r+s}}\right] \otimes 1+1 \otimes\left[S_{h_{r+s}}\right]\right)
$$

exactly $h t(U)$ factors, recorded by $\mathbf{j}_{1}$, of the form $\left[S_{h_{k}}\right] \otimes 1$ and $h t(W)$ factors, recorded by $\mathbf{j}_{2}$, of the form $1 \otimes\left[S_{h_{k}}\right]$. With this notation we are looking at the products $\left[S_{j_{1}}\right] \cdots\left[S_{j_{t}}\right] \otimes$ $\left[S_{j_{t+1}}\right] \cdots\left[S_{j_{r+s}}\right]$, where again we have used the abbreviation $\mathrm{ht}(U)=t$. The first and second sums in $E$ as well as the flag coefficients should now be transparent. In order to record the contribution of the twisted multiplication on $\mathcal{H}(Q, \mathbf{d}) \otimes \mathcal{H}(Q, \mathbf{d})$ to the coefficient of $[U] \otimes[W]$ 
we need a shuffle $\sigma \in \Sigma_{\mathrm{ht}(U), \mathrm{ht}(W)}$ and $j_{k}=h_{\sigma_{k}^{-1}}$. Indeed, the condition $\sigma_{k}^{-1}>\sigma_{\ell}^{-1}$ of the last sum in $E$ records a product of the form $\left(1 \otimes\left[S_{j_{k}}\right]\right)\left(\left[S_{j_{\ell}}\right] \otimes 1\right)$ and thus produces the stated contribution.

As previously stated, Theorem 5.4 allows us, for a fixed $[U] \otimes[W]$, to extract the equality below from which the claim will follow:

$$
\begin{gathered}
\sum_{[V]} v^{\langle|U|,|W|\rangle+\sum_{k<\ell}\left\langle\alpha_{h_{k}}, \alpha_{h_{\ell}}\right\rangle} \frac{|\operatorname{Aut}(U)||\operatorname{Aut}(W)|}{|\operatorname{Aut}(V)|}\left|\mathcal{F}_{U W}^{V}\right|\left|\mathcal{F}_{\mathbf{h}}(V)\right| \\
=\sum_{\substack{\sigma \in \Sigma_{\mathbf{h t}(U), \operatorname{ht}(W)} \\
\mathbf{j}_{1}, \mathbf{j}_{2} \in \mathbf{W}: \mathbf{j}_{\sigma}=\mathbf{h}}} v^{E}\left|\mathcal{F}_{\mathbf{j}_{1}}(U)\right|\left|\mathcal{F}_{\mathbf{j}_{2}}(W)\right| .
\end{gathered}
$$

To complete the proof we perform a few simple manipulations with the sums appearing in the exponents of (7.3) and (7.4). To start we match the left hand sides of (7.3) and (7.4) by multiplying both sides of (7.3) by $v^{\sum_{k<\ell}\left\langle\alpha_{h_{\ell}}, \alpha_{h_{k}}\right\rangle-\frac{1}{2}\langle|U|,|W|\rangle-\frac{1}{2}\langle|W|,|U|\rangle}$ and both sides of (7.4) by $v^{-\sum_{k<\ell}\left\langle\alpha_{h_{k}}, \alpha_{h_{\ell}}\right\rangle}$. Thus it remains to establish the following identity of exponents.

Lemma 7.4. For any $\sigma \in \Sigma_{\mathrm{ht}(U), \mathrm{ht}(W)}$ and words $\mathbf{j}_{1}, \mathbf{j}_{2}$ satisfying $\mathcal{F}_{\mathbf{j}_{1}}(U) \neq \varnothing, \mathcal{F}_{\mathbf{j}_{2}}(W) \neq \varnothing$, and $\mathbf{j}_{\sigma}=\mathbf{h}$ we have:

$$
\begin{aligned}
& \sum_{k<\ell}\left\langle\alpha_{h_{\ell}}, \alpha_{h_{k}}\right\rangle-\sum_{1 \leq k<\ell \leq t}\left\langle\alpha_{j_{\ell}}, \alpha_{j_{k}}\right\rangle-\sum_{t+1 \leq k<\ell \leq r+s}\left\langle\alpha_{j_{\ell}}, \alpha_{j_{k}}\right\rangle+\zeta(\sigma)-\frac{1}{2}\langle|U|,|W|\rangle-\frac{1}{2}\langle|W|,|U|\rangle \\
& =-\sum_{k<\ell}\left\langle\alpha_{h_{k}}, \alpha_{h_{\ell}}\right\rangle+\sum_{1 \leq k<\ell \leq t}\left\langle\alpha_{j_{k}}, \alpha_{j_{\ell}}\right\rangle+\sum_{\substack{t+1 \leq k<\ell \leq r+s\\
}}\left\langle\alpha_{j_{k}}, \alpha_{j_{\ell}}\right\rangle+\sum_{\substack{1 \leq k \leq t \\
t+1 \leq \ell \leq r+s \\
\sigma_{k}^{-1}>\sigma_{\ell}^{-1}}}\left(\alpha_{j_{k}}, \alpha_{j_{\ell}}\right) .
\end{aligned}
$$

Proof. We first eliminate the dependence on $\mathbf{h}$ using the following identities which are immediate consequences of the equality $j_{k}=h_{\sigma_{k}^{-1}}$ :

$$
\begin{aligned}
& \sum_{k<\ell}\left\langle\alpha_{h_{\ell}}, \alpha_{h_{k}}\right\rangle-\sum_{1 \leq k<\ell \leq t}\left\langle\alpha_{j_{\ell}}, \alpha_{j_{k}}\right\rangle-\sum_{t+1 \leq k<\ell \leq r+s}\left\langle\alpha_{j_{\ell}}, \alpha_{j_{k}}\right\rangle \\
& =\sum_{\substack{1 \leq k \leq t \\
t+1 \leq \ell \leq r+s \\
\sigma_{k}^{-1}<\sigma_{\ell}^{-1}}}\left\langle\alpha_{j_{\ell}}, \alpha_{j_{k}}\right\rangle+\sum_{\substack{1 \leq k \leq t \\
t+1 \leq \ell \leq r+s \\
\sigma_{\ell}^{-1}<\sigma_{k}^{-1}}}\left\langle\alpha_{j_{k}}, \alpha_{j_{\ell}}\right\rangle, \\
& -\sum_{k<\ell}\left\langle\alpha_{h_{k}}, \alpha_{h_{\ell}}\right\rangle+\sum_{1 \leq k<\ell \leq t}\left\langle\alpha_{j_{k}}, \alpha_{j_{\ell}}\right\rangle+\sum_{t+1 \leq k<\ell \leq r+s}\left\langle\alpha_{j_{k}}, \alpha_{j_{\ell}}\right\rangle \\
& =-\sum_{\substack{1 \leq k \leq t \\
t+1 \leq \ell \leq r+s \\
\sigma_{k}^{-1}<\sigma_{\ell}^{-1}}}\left\langle\alpha_{j_{k}}, \alpha_{j_{\ell}}\right\rangle-\sum_{\substack{1 \leq k \leq t \\
t+1 \leq \ell \leq r+s \\
\sigma_{\ell}^{-1}<\sigma_{k}^{-1}}}\left\langle\alpha_{j_{\ell}}, \alpha_{j_{k}}\right\rangle,
\end{aligned}
$$

this reduces (7.5) to showing

$$
\begin{gathered}
\left.\sum_{\substack{1 \leq k \leq t \\
t+1 \leq \ell \leq r+s \\
\sigma_{k}^{-1}<\sigma_{\ell}^{-1}}}\left\langle\alpha_{j_{\ell}}, \alpha_{j_{k}}\right\rangle+\sum_{\substack{1 \leq k \leq t \\
t+1 \leq \ell \leq r+s \\
\sigma_{\ell}^{-1}<\sigma_{k}^{-1}}}\left\langle\alpha_{j_{k}}, \alpha_{j_{\ell}}\right\rangle+\zeta(\sigma)-\frac{1}{2}\langle|U|,|W|\rangle-\frac{1}{2}\langle|W|,|U|\rangle\right] \\
=-\sum_{\substack{1 \leq k \leq t \\
t+1 \leq \ell \leq r+s \\
\sigma_{k}^{-1}<\sigma_{\ell}^{-1}}}\left\langle\alpha_{j_{k}}, \alpha_{j_{\ell}}\right\rangle-\sum_{\substack{1 \leq k \leq t \\
t+1 \leq \ell \leq r+s \\
\sigma_{\ell}^{-1}<\sigma_{k}^{-1}}}\left\langle\alpha_{j_{\ell}}, \alpha_{j_{k}}\right\rangle+\sum_{\substack{1 \leq k \leq t \\
t+1 \leq \ell \leq r+s \\
\sigma_{k}^{-1}>\sigma_{\ell}^{-1}}}\left(\alpha_{j_{k}}, \alpha_{j_{\ell}}\right) .
\end{gathered}
$$


Using the dichotomy $\sigma_{k}^{-1}<\sigma_{\ell}^{-1}$ or $\sigma_{\ell}^{-1}<\sigma_{k}^{-1}$ for $1 \leq k \leq t$ and $t+1 \leq \ell \leq r+s$ the last sum on the right hand side of (7.6) becomes:

$$
\sum_{\substack{1 \leq k \leq t \\ t+1 \leq \ell \leq r+s \\ \sigma_{k}^{-1}>\sigma_{\ell}^{-1}}}\left(\alpha_{j_{k}}, \alpha_{j_{\ell}}\right)=\zeta(\sigma)+\frac{1}{2} \sum_{\substack{1 \leq k \leq t \\ t+1 \leq \ell \leq r+s}}\left(\alpha_{j_{k}}, \alpha_{j_{\ell}}\right) .
$$

Rewriting $\left(\alpha_{j_{k}}, \alpha_{j_{\ell}}\right)=\left\langle\alpha_{j_{k}}, \alpha_{j_{\ell}}\right\rangle+\left\langle\alpha_{j_{\ell}}, \alpha_{j_{k}}\right\rangle$ and applying (7.7) in the right hand side of (7.6) gives:

$$
\begin{aligned}
& -\sum_{\substack{1 \leq k \leq t \\
t+1 \leq \ell \leq r+s \\
\sigma_{k}^{-1}<\sigma_{\ell}^{-1}}}\left\langle\alpha_{j_{k}}, \alpha_{j_{\ell}}\right\rangle-\sum_{\substack{1 \leq k \leq t \\
t+1 \leq \ell \leq r+s \\
\sigma_{\ell}^{-1}<\sigma_{k}^{-1}}}\left\langle\alpha_{j_{\ell}}, \alpha_{j_{k}}\right\rangle+\zeta(\sigma)+\frac{1}{2} \sum_{\substack{1 \leq k \leq t \\
t+1 \leq \ell \leq r+s}}\left(\left\langle\alpha_{j_{k}}, \alpha_{j_{\ell}}\right\rangle+\left\langle\alpha_{j_{\ell}}, \alpha_{j_{k}}\right\rangle\right) \\
& =-\frac{1}{2} \sum_{\substack{1 \leq k \leq t \\
t+1 \leq \ell \leq r+s}}\left(\left\langle\alpha_{j_{k}}, \alpha_{j_{\ell}}\right\rangle+\left\langle\alpha_{j_{\ell}}, \alpha_{j_{k}}\right\rangle\right)+\zeta(\sigma) \\
& +\sum_{\substack{1 \leq k \leq t \\
t+1 \leq \ell \leq r+s \\
\sigma_{k}^{-1}<\sigma_{\ell}^{-1}}}\left\langle\alpha_{j_{\ell}}, \alpha_{j_{k}}\right\rangle+\sum_{\substack{1 \leq k \leq t \\
t+1 \leq \ell \leq r+s \\
\sigma_{\ell}^{-1}<\sigma_{k}^{-1}}}\left\langle\alpha_{j_{k}}, \alpha_{j_{\ell}}\right\rangle \\
& =-\frac{1}{2}\langle|U|,|W|\rangle-\frac{1}{2}\langle|W|,|U|\rangle+\zeta(\sigma)+\sum_{\substack{1 \leq k \leq t \\
t+1 \leq \ell \leq r+s \\
\sigma_{k}^{-1}<\sigma_{\ell}^{-1}}}\left\langle\alpha_{j_{\ell}}, \alpha_{j_{k}}\right\rangle+\sum_{\substack{1 \leq k \leq t \\
t+1 \leq \ell \leq r+s \\
\sigma_{\ell}^{-1}<\sigma_{k}^{-1}}}\left\langle\alpha_{j_{k}}, \alpha_{j_{\ell}}\right\rangle
\end{aligned}
$$

as desired, which completes the proof of Lemma 7.4.

This completes the proof of Lemma 7.3.

With Lemma 7.3 proven, we may conclude that $\Omega$ defines a homomorphism of algebras. It remains to show that $\Delta \Omega\left([V]^{*}\right)=(\Omega \otimes \Omega)\left(\Delta\left([V]^{*}\right)\right)$. The coproduct $\Delta\left([V]^{*}\right)$ can be expanded in the same rescaled basis as

$$
\begin{aligned}
\Delta\left([V]^{*}\right) & =v^{-\frac{1}{2}\langle|V|,|V|\rangle+\frac{1}{2} \sum_{i=1}^{n} d_{i} v_{i}} \Delta\left(\delta_{[V]}\right) \\
& =\sum_{[U],[W]} v^{-\frac{1}{2}\langle|U|+|W|,|U|+|W|\rangle+\frac{1}{2} \sum_{i=1}^{n} d_{i}\left(u_{i}+w_{i}\right)} v^{\langle|U|,|W|\rangle\left|\mathcal{F}_{U W}^{V}\right| \cdot \delta_{[U]} \otimes \delta_{[W]}} \\
& =\sum_{[V]} v^{\frac{1}{2}\langle|U|,|W|\rangle-\frac{1}{2}\langle|W|,|U|\rangle}\left|\mathcal{F}_{U W}^{V}\right| \cdot[U]^{*} \otimes[W]^{*} .
\end{aligned}
$$

Then the identity $\Delta \Omega\left([V]^{*}\right)=(\Omega \otimes \Omega)\left(\Delta\left([V]^{*}\right)\right)$ is equivalent to the following collection of identities involving flags.

Lemma 7.5. For all words $\mathbf{j}_{1}, \mathbf{j}_{2} \in \mathbf{W}$ we have

$$
\begin{gathered}
v^{-\sum_{k<\ell}\left\langle\alpha_{h_{\ell}}, \alpha_{h_{k}}\right\rangle} v^{\frac{1}{2}\left(\left|\mathbf{j}_{1}\right|,\left|\mathbf{j}_{2}\right|\right)}\left|\mathcal{F}_{\mathbf{h}}(V)\right|=\sum_{[U],[W]} v^{-\sum_{1 \leq k<\ell \leq r}\left\langle\alpha_{j_{\ell}}, \alpha_{j_{k}}\right\rangle} v^{-\sum_{r+1 \leq k<\ell \leq r+s}\left\langle\alpha_{j_{\ell}}, \alpha_{j_{k}}\right\rangle} \\
\times v^{\frac{1}{2}\langle|U|,|W|\rangle-\frac{1}{2}\langle|W|,|U|\rangle\left|\mathcal{F}_{U, W}^{V}\right| \cdot\left|\mathcal{F}_{\mathbf{j}_{1}}(U)\right| \cdot\left|\mathcal{F}_{\mathbf{j}_{2}}(W)\right|}
\end{gathered}
$$

where we used the word $\mathbf{h}=\left(\mathbf{j}_{1}, \mathbf{j}_{2}\right)$. 
Proof. This essentially follows from the associativity of multiplication in the Hall-Ringel algebra $\mathcal{H}(Q, \mathbf{d})$. Indeed, expanding the products

$$
\left[S_{h_{1}}\right] \cdots\left[S_{h_{r+s}}\right]=\left(\left[S_{j_{1}}\right] \cdots\left[S_{j_{r}}\right]\right) \cdot\left(\left[S_{j_{r+1}}\right] \cdots\left[S_{j_{r+s}}\right]\right)
$$

gives $\left|\mathcal{F}_{\mathbf{h}}(V)\right|=\left|\mathcal{F}_{U, W}^{V}\right| \cdot\left|\mathcal{F}_{\mathbf{j}_{1}}(U)\right| \cdot\left|\mathcal{F}_{\mathbf{j}_{2}}(W)\right|$. To see the equality of the exponents on either side we note that

$$
\frac{1}{2}\left(\mathbf{j}_{1}, \mathbf{j}_{2}\right)=\frac{1}{2}\left\langle\left|\mathbf{j}_{1}\right|,\left|\mathbf{j}_{2}\right|\right\rangle+\frac{1}{2}\left\langle\left|\mathbf{j}_{2}\right|,\left|\mathbf{j}_{1}\right|\right\rangle=\frac{1}{2}\langle|U|,|W|\rangle+\frac{1}{2}\langle|W|,|U|\rangle .
$$

Combining this with the equality

$$
-\sum_{k<\ell}\left\langle\alpha_{h_{\ell}}, \alpha_{h_{k}}\right\rangle=-\sum_{1 \leq k<\ell \leq r}\left\langle\alpha_{j_{\ell}}, \alpha_{j_{k}}\right\rangle-\sum_{r+1 \leq k<\ell \leq r+s}\left\langle\alpha_{j_{\ell}}, \alpha_{j_{k}}\right\rangle-\left\langle\left|\mathbf{j}_{2}\right|,\left|\mathbf{j}_{1}\right|\right\rangle
$$

completes the proof.

To finish the proof of Theorem 7.1 we note that $\Omega$ respects the grading and thus, since the multiplication on both $\mathcal{H}^{*}(Q, \mathbf{d}) \otimes \mathcal{H}^{*}(Q, \mathbf{d})$ and $g^{*} \otimes g^{*}$ are twisted by the grading, we may conclude that $\Omega$ is a homomorphism of twisted bialgebras.

We now show that this definition of $\Omega$ does in fact make the tetrahedron commute. The left hand triangle is easily seen to be commutative by looking at generators of $\mathcal{A}_{v}[N]$. Thus we focus on the right hand triangle.

Theorem 7.6. There is an equality of homomorphisms $\widetilde{\Psi}_{\mathbf{i}}=\bar{\Psi}_{\mathbf{i}} \circ \Omega$.

Proof. It suffices to check the equality on the basis $[V]^{*}$ of $\mathcal{H}^{*}(Q, \mathbf{d})$. Indeed, the value of $\widetilde{\Psi}_{\mathbf{i}}$ on $[V]^{*}$ is provided by Theorem 5.7:

$$
\widetilde{\Psi}_{\mathbf{i}}\left([V]^{*}\right)=\sum_{\mathbf{a} \in \mathbb{Z}_{\geq 0}^{m}} v^{-\sum_{k<\ell} a_{k} a_{\ell}\left\langle\alpha_{i_{\ell}}, \alpha_{i_{k}}\right\rangle}\left|\mathcal{F}_{\mathbf{i}, \mathbf{a}}(V)\right| t^{\mathbf{a}} .
$$

Observe that the passage from $\mathcal{F}_{\mathbf{i}, \mathbf{a}}(V)$ to $\mathcal{F}_{\mathbf{i}^{\mathbf{a}}}(V)$ amounts to choosing a basis in each quotient $S_{i_{k}}^{a_{k}}$ for which there are $\left(a_{k}\right)_{i_{k}}$ possibilities. Thus we have the identity:

$$
\begin{aligned}
\widetilde{\Psi}_{\mathbf{i}}\left([V]^{*}\right) & =\sum_{\mathbf{a} \in \mathbb{Z}_{\geq 0}^{m}} v^{-\sum_{k<\ell} a_{k} a_{\ell}\left\langle\alpha_{i_{\ell}}, \alpha_{i_{k}}\right\rangle}\left|\mathcal{F}_{\mathbf{i}^{\mathbf{a}}}(V)\right| \frac{1}{\left.\left(a_{1}\right)\right)_{i_{1}}^{!} \cdots\left(a_{m}\right) !} t_{i_{m}}^{\mathbf{a}} \\
& =\sum_{\mathbf{a} \in \mathbb{Z}_{\geq 0}^{m}} v^{-\sum_{k<\ell} a_{k} a_{\ell}\left\langle\alpha_{i_{\ell}}, \alpha_{i_{k}}\right\rangle}\left|\mathcal{F}_{\mathbf{i}^{\mathbf{a}}}(V)\right| \frac{v^{-\sum_{k=1}^{m} d_{i_{k}}\left(\begin{array}{c}
a_{k} \\
2
\end{array}\right)}}{\left[a_{1}\right]_{i_{1}}^{!} \cdots\left[a_{m}\right]_{i_{m}}^{!}} t^{\mathbf{a}},
\end{aligned}
$$

where we passed to the bar invariant $q$-numbers in (7.8).

Now working from the definitions of $\Omega$ and $\bar{\Psi}_{\mathbf{i}}$ we get:

$$
\bar{\Psi}_{\mathbf{i}} \circ \Omega\left([V]^{*}\right)=\sum_{\mathbf{a} \in \mathbb{Z}_{\geq 0}^{m}} v^{-\sum_{k<\ell}\left\langle\alpha_{j_{\ell}}, \alpha_{j_{k}}\right\rangle}\left|\mathcal{F}_{\mathbf{i}^{\mathbf{a}}}(V)\right| \frac{1}{\left[a_{1}\right]_{i_{1}}^{!} \cdots\left[a_{m}\right]_{i_{m}}^{!}} t^{\mathbf{a}},
$$

where we have used $\mathbf{j}=\mathbf{i}^{\mathbf{a}}=(\underbrace{i_{1}, \ldots, i_{1}}_{a_{1}}, \ldots, \underbrace{i_{m}, \ldots, i_{m}}_{a_{m}})$ in the exponent of $v$ in (7.9). But notice that

$$
\sum_{k<\ell}\left\langle\alpha_{j_{\ell}}, \alpha_{j_{k}}\right\rangle=d_{i_{k}}\left(\begin{array}{c}
a_{k} \\
2
\end{array}\right)+\sum_{k<\ell} a_{k} a_{\ell}\left\langle\alpha_{i_{\ell}}, \alpha_{i_{k}}\right\rangle
$$


where $d_{i_{k}}\left(\begin{array}{c}a_{k} \\ 2\end{array}\right)$ comes from taking $j_{k}$ and $j_{\ell}$ from within a single grouping $\underbrace{i_{k}, \ldots, i_{k}}_{a_{k}}$ while $\sum_{k<\ell} a_{k} a_{\ell}\left\langle\alpha_{i_{\ell}}, \alpha_{i_{k}}\right\rangle$ is obtained by taking $j_{k}$ and $j_{\ell}$ from different groupings. Thus we see that $\widetilde{\Psi}_{\mathbf{i}}\left([V]^{*}\right)=\bar{\Psi}_{\mathbf{i}} \circ \Omega\left([V]^{*}\right)$ for any representation $V \in \operatorname{rep}_{\mathbb{F}}(Q, \mathbf{d})$.

In fact, there is an extra bit of structure preserved by the quantum shuffle character $\Omega$.

\section{Proposition 7.7.}

(a) The quantum shuffle character $\Omega$ intertwines the action of $\theta_{S_{i}}$ on $\mathcal{H}^{*}(Q, \mathbf{d})$ and the action of $\theta_{i}$ on $g^{*}$, i.e. $\theta_{i} \circ \Omega=\Omega \circ \theta_{S_{i}}$.

(b) The quantum shuffle character $\Omega$ intertwines the action of $\theta_{S_{i}}^{*}$ on $\mathcal{H}^{*}(Q, \mathbf{d})$ and the action of $\theta_{i}^{*}$ on $g^{*}$, i.e. $\theta_{i}^{*} \circ \Omega=\Omega \circ \theta_{S_{i}}^{*}$.

Proof. To prove (a) we simply compute the actions on a basis vector $[V]^{*}$ :

$$
\begin{aligned}
\Omega \circ \theta_{S_{i}}\left([V]^{*}\right) & =v^{-\frac{1}{2}\langle V, V\rangle+\frac{1}{2} \sum_{i=1}^{n} d_{i} v_{i}} \Omega \circ \theta_{S_{i}}\left(\delta_{[V]}\right) \\
& =v^{-\frac{1}{2}\langle V, V\rangle+\frac{1}{2} \sum_{i=1}^{n} d_{i} v_{i}} \sum_{[W]} v^{\left\langle|W|, \alpha_{i}\right\rangle}\left|\mathcal{F}_{W S_{i}}^{V}\right| \cdot \Omega\left(\delta_{[W]}\right) \\
& =\sum_{[W]} v^{\frac{1}{2}\left\langle W, \alpha_{i}\right\rangle-\frac{1}{2}\left\langle\alpha_{i}, W\right\rangle}\left|\mathcal{F}_{W S_{i}}^{V}\right| \cdot \Omega\left([W]^{*}\right) \\
& =\sum_{[W]} \sum_{\mathbf{j} \in \mathbf{W}} v^{\frac{1}{2}\left\langle W, \alpha_{i}\right\rangle-\frac{1}{2}\left\langle\alpha_{i}, W\right\rangle} v^{-\sum_{k<\ell}\left\langle\alpha_{j_{\ell}}, \alpha_{j_{k}}\right\rangle}\left|\mathcal{F}_{W S_{i}}^{V}\right| \cdot\left|\mathcal{F}_{\mathbf{j}}(W)\right| \cdot \mathbf{j} \\
& =\sum_{\mathbf{j} \in \mathbf{W}} v^{\frac{1}{2}\left(|\mathbf{j}|, \alpha_{i}\right)-\left\langle\alpha_{i},|\mathbf{j}|\right\rangle} v^{-\sum_{k<\ell}\left\langle\alpha_{j_{\ell}}, \alpha_{j_{k}}\right\rangle}\left|\mathcal{F}_{(\mathbf{j}, i)}(V)\right| \cdot \mathbf{j},
\end{aligned}
$$

where we have used in the last equality that $\sum_{[W]}\left|\mathcal{F}_{W S_{i}}^{V}\right| \cdot\left|\mathcal{F}_{\mathbf{j}}(W)\right|=\left|\mathcal{F}_{(\mathbf{j}, i)}(V)\right|$ and $|W|=|\mathbf{j}|$ whenever $\mathcal{F}_{\mathbf{j}}(W) \neq \varnothing$. On the other hand we have:

$$
\theta_{i} \circ \Omega\left([V]^{*}\right)=\sum_{\mathbf{j} \in \mathbf{W}} v^{-\sum_{k<\ell}\left\langle\alpha_{j_{\ell}}, \alpha_{j_{k}}\right\rangle}\left|\mathcal{F}_{\mathbf{j}}(V)\right| \cdot \theta_{i}(\mathbf{j}) .
$$

A simple inspection using that $\theta_{i}(\mathbf{j})=0$ unless $\mathbf{j}$ ends with the letter $i$ shows that equations (7.10) and (7.11) agree. The proof of (b) is similar.

Our main conjecture of this note is that the quantum shuffle character $\Omega$ relates the representation theory of $(Q, \mathbf{d})$ with the representation theory of KLR (quiver Hecke) algebras. We will present necessary background and our conjectures in the next section. First we draw a useful conclusion from the existence of the homomorphism $\Omega$.

Corollary 7.8. For any rigid representation $V$ of $(Q, \mathbf{d})$ and word $\mathbf{j}$, the variety $\mathcal{F}_{\mathbf{j}}(V)$ has a counting polynomial.

Proof. Let $V$ be a rigid representation of $(Q, \mathbf{d})$ over a finite field $\mathbb{F}$ and write $\nu \in \mathcal{Q}$ for the dimension vector of $V$, a positive root in $\Phi$. Since $V$ is rigid, $[V]^{*} \in \mathcal{A}_{v}[N] \subset \mathcal{H}^{*}(Q, \mathbf{d})$, where $v=\sqrt{|\mathbb{F}|}$. In fact, following [5] and [11] there exists a recursively computable element $x_{\nu} \in \mathcal{A}_{v}[N]$, where $v$ is a formal parameter, which specializes to $[V]^{*}$ under the map $v \mapsto \sqrt{|\mathbb{F}|}$. Since the recursion computing $x_{\nu}$ is described purely in terms of the root datum, for an arbitrary 
finite field $\mathbb{F}^{\prime}$ the same element $x_{\nu}$ specializes under the map $v \mapsto \sqrt{\left|\mathbb{F}^{\prime}\right|}$ to the class of the unique indecomposable rigid representation of $(Q, \mathbf{d})$ over $\mathbb{F}^{\prime}$ with dimension vector $\nu$.

We may alternatively consider the image of $x_{\nu}$ under the embedding $\iota: \mathcal{A}_{v}[N] \rightarrow g^{*}$. By the commutativity of the left hand triangle in $(7.1), \iota\left(x_{\nu}\right)$ specializes to $\Omega\left([V]^{*}\right)$ under the map $v \mapsto \sqrt{|\mathbb{F}|}$. Now recalling the definition of $\Omega\left([V]^{*}\right)$ we see that (up to a power of $v$ ) the counting polynomial of $\mathcal{F}_{\mathbf{j}}(V)$ is the coefficient of $\mathbf{j}$ in $\iota\left(x_{\nu}\right)$. As above, this polynomial is independent of the choice of specialization.

\section{KLR algebras}

Recall the root lattice $\mathcal{Q}$ associated to the symmetrizable $I \times I$ Cartan matrix $A$ with symmetrizers $\mathbf{d}=\left(d_{i}: i \in I\right)$. Write $\mathcal{Q}_{+}=\bigoplus_{i \in I} \mathbb{Z}_{\geq 0} \alpha_{i}$ for the positive cone in $\mathcal{Q}$ generated by the simple roots $\left\{\alpha_{i}\right\}_{i \in I}$. The KLR (quiver Hecke) algebra $R=\bigoplus_{\nu \in \mathcal{Q}_{+}} R_{\nu}$ is defined diagrammatically by Khovanov-Lauda $[14,15]$ and symbolically in the independent work of Rouquier [32, 33]. We follow the symbolic approach as presented in [19].

Let $\mathbb{k}$ denote any field of characteristic zero. The relations in $R_{\nu}$ will depend on the polynomials

$$
Q_{i j}(u, v):= \begin{cases}0 & \text { if } i=j, \\ 1 & \text { if } a_{i j}=0 \\ \varepsilon_{i j}\left(u^{-a_{i j}}-v^{-a_{j i}}\right) & \text { if } a_{i j}<0\end{cases}
$$

where, for simple representations $S_{i}$ and $S_{j}$ of $(Q, \mathbf{d})$, we set $\varepsilon_{i j}=1=-\varepsilon_{j i}$ if $\operatorname{Ext}^{1}\left(S_{i}, S_{j}\right) \neq 0$ and $\varepsilon_{i j}=0$ if $\operatorname{Ext}^{1}\left(S_{i}, S_{j}\right)=\operatorname{Ext}^{1}\left(S_{j}, S_{i}\right)=0$. Suppose the sum of the entries in $\nu$ is $d$. Then the $K L R$ (quiver Hecke) algebra $R_{\nu}$ is the $\mathbb{k}$-algebra with polynomial generators $y_{1}, \ldots, y_{d}$, braid-like generators $\psi_{1}, \ldots, \psi_{d-1}$, and orthogonal idempotents $e_{\mathbf{j}}$, where $\mathbf{j} \in \mathbf{W}^{\nu}$ ranges over words with $|\mathbf{j}|=\nu$ (note that all such words have length $d$ ). These generators satisfy the following relations:

$$
\begin{aligned}
& \sum_{\mathbf{j} \in \mathbf{W}^{\nu}} e_{\mathbf{j}}=1_{\nu}, \quad y_{k} e_{\mathbf{j}}=e_{\mathbf{j}} y_{k}, \quad y_{k} y_{\ell}=y_{\ell} y_{k}, \\
& \psi_{k} e_{\mathbf{j}}=e_{\sigma_{k} \mathbf{j}} \psi_{k}, \quad\left(y_{k} \psi_{\ell}-\psi_{\ell} y_{\sigma_{\ell}(k)}\right) e_{\mathbf{j}}=\delta_{j_{\ell}, j_{\ell+1}}\left(\delta_{k, \ell+1}-\delta_{k, \ell}\right) e_{\mathbf{j}}, \\
& \psi_{k}^{2} e_{\mathbf{j}}=Q_{j_{k}, j_{k+1}}\left(y_{k}, y_{k+1}\right) e_{\mathbf{j}}, \quad \psi_{k} \psi_{\ell}=\psi_{\ell} \psi_{k} \text { for }|k-\ell|>1, \\
& \left(\psi_{k+1} \psi_{k} \psi_{k+1}-\psi_{k} \psi_{k+1} \psi_{k}\right) e_{\mathbf{j}}=\delta_{j_{k}, j_{k+2}} \frac{Q_{j_{k}, j_{k+1}}\left(y_{k+2}, y_{k+1}\right)-Q_{j_{k}, j_{k+1}}\left(y_{k}, y_{k+1}\right)}{y_{k+2}-y_{k}} e_{\mathbf{j}}
\end{aligned}
$$

The KLR algebra $R_{\nu}$ admits a $\mathbb{Z}$-grading given by

$$
\operatorname{deg}\left(e_{\mathbf{j}}\right)=0, \quad \operatorname{deg}\left(y_{k} e_{\mathbf{j}}\right)=2 d_{j_{k}}, \quad \operatorname{deg}\left(\psi_{k} e_{\mathbf{j}}\right)=-d_{j_{k}} a_{j_{k} j_{k+1}} .
$$

Consider the category $R-\bmod$ of all finite-dimensional graded $R$-modules. This category decomposes into the blocks $R_{\nu}-\bmod \left(\nu \in \mathcal{Q}_{+}\right)$consisting of finite-dimensional graded $R_{\nu^{-}}$ modules.

Using the natural embeddings $R_{\nu} \otimes R_{\mu} \hookrightarrow R_{\nu+\mu}$ we get induction and restriction functors

$$
\begin{array}{ll}
\operatorname{Ind}_{\nu, \mu}^{\nu+\mu}: & R_{\nu} \otimes R_{\mu}-\bmod \rightarrow R_{\nu+\mu}-\bmod , \\
\operatorname{Res}_{\nu, \mu}^{\nu+\mu}: & R_{\nu+\mu}-\bmod \rightarrow R_{\nu} \otimes R_{\mu}-\bmod .
\end{array}
$$

These are adjoint in the following sense:

$$
\operatorname{Hom}_{R_{\nu+\mu}}\left(\operatorname{Ind}_{\nu, \mu}^{\nu+\mu} M, N\right) \cong \operatorname{Hom}_{R_{\nu} \otimes R_{\mu}}\left(M, \operatorname{Res}_{\nu, \mu}^{\nu+\mu} N\right)
$$

for every $M \in R_{\nu} \otimes R_{\mu}-\bmod$ and $N \in R_{\nu+\mu}-\bmod$. 
For $\nu, \mu \in \mathcal{Q}_{+}$and $M \in R_{\nu}-\bmod , N \in R_{\mu}-\bmod$ we define $M \circ N \in R_{\nu+\mu}-\bmod$ by

$$
M \circ N=\operatorname{Ind}_{\nu, \mu}^{\nu+\mu}(M \otimes N) .
$$

With this product the Grothendieck group $\mathcal{K}(R-\bmod )=\bigoplus_{\nu \in \mathcal{Q}_{+}} \mathcal{K}\left(R_{\nu}-\bmod \right)$ generated by isomorphism classes of finite dimensional graded $R$-modules has the structure of an associative unital ring. The identity element is given by the isomorphism class of $R_{0}$ thought of as an $R_{0^{-}}$ module and multiplication is given by $[M][N]=[M \circ N]$ for $M \in R_{\nu}-\bmod$ and $N \in R_{\mu}-\bmod$.

For a finite dimensional graded vector space $V=\bigoplus_{n \in \mathbb{Z}} V_{n}$ write $\operatorname{dim}_{v} V=\sum_{n \in \mathbb{Z}}\left(\operatorname{dim} V_{n}\right) v^{n}$ for its graded character. The algebra $R$ is graded and thus its Grothendieck ring admits an action of $\mathbb{Z}\left[v^{ \pm 1}\right]$, where $v$ acts by grading shift. There is a $\mathbb{Z}\left[v^{ \pm 1}\right]$-linear character map $\mathrm{ch}_{v}$ from $\mathcal{K}(R-\bmod )$ to the quantum shuffle algebra $g^{*}$ given by

$$
[M] \mapsto \operatorname{ch}_{v}(M):=\sum_{\mathbf{j} \in \mathbf{W}} \operatorname{dim}_{v} M_{\mathbf{j}} \cdot \mathbf{j},
$$

where $M_{\mathbf{j}}=e_{\mathbf{j}} M$ and the sum is finite since $M$ is a finite-dimensional representation. The following theorem provides the monoidal categorification of quantum groups.

Theorem $8.1([15,33,37])$. The $\operatorname{map} \mathrm{ch}_{v}: \mathcal{K}(R-\bmod ) \rightarrow g^{*}$ is an injective algebra homomorphism, i.e.

$$
\operatorname{ch}_{v}(M \circ N)=\operatorname{ch}_{v}(M) \circ \operatorname{ch}_{v}(N),
$$

and its image is precisely $\mathcal{A}_{v}[N]$. Moreover, in symmetric types the irreducible characters correspond to elements of the dual canonical basis.

Thus it is natural that a great deal of effort has been put forth to describe and construct the irreducible representations of KLR algebras. Currently the main approach is to realize them as the irreducible heads of certain "standard modules" which admit a combinatorial description. This approach has been pursued by Kleshchev-Ram [19], McNamara [27], and Benkart-KangOh-Park [1] in finite types and by Kleshchev [17] in the affine case. An inductive approach to classifying irreducible $R$-modules has been suggested by Lauda-Vazirani in [22]. We propose an entirely new construction of characters for certain irreducible $R$-modules using the representation theory of $(Q, \mathbf{d})$ and the quantum shuffle character.

Conjecture 8.2. For any rigid representation $V$ of $(Q, \mathbf{d})$, the quantum shuffle character $\Omega\left([V]^{*}\right)$ produces the character of an irreducible KLR representation.

The following example provides some small evidence for Conjecture 8.2 for arbitrary valued quivers $(Q, \mathbf{d})$.

Example 8.3. It is shown in [22, Theorem 6.10] that for $\nu=-a_{i j} \alpha_{i}+\alpha_{j}(i \neq j)$ the block $R_{\nu}-\bmod$ contains a unique irreducible module $L\left(i^{-a_{i j}}, j\right)$ with $\operatorname{ch}_{v}\left(L\left(i^{-a_{i j}}, j\right)\right)=\left[-a_{i j}\right]_{i}^{!}\left(i^{-a_{i j}}, j\right)$ and a unique irreducible module $L\left(j, i^{-a_{i j}}\right)$ with $\operatorname{ch}_{v}\left(L\left(j, i^{-a_{i j}}\right)\right)=\left[-a_{i j}\right]_{i}^{!}\left(j, i^{-a_{i j}}\right)$.

Now consider the full subquiver $\left(Q_{i j},\left(d_{i}, d_{j}\right)\right)$ of $(Q, \mathbf{d})$ on vertices $i$ and $j$ (without loss of generality assume the arrows point from $i$ to $j)$. Any representation of $\left(Q_{i j},\left(d_{i}, d_{j}\right)\right)$ is automatically a representation of $(Q, \mathbf{d})$ and rigidity is carried through this equivalence. The quiver $\left(Q_{i j},\left(d_{i}, d_{j}\right)\right)$ has a unique non-simple injective representation $I_{j}$, the injective hull of the simple $S_{j}$, and its dimension vector is precisely $-a_{i j} \alpha_{i}+\alpha_{j}$. We aim to show that $\Omega\left(\left[I_{j}\right]^{*}\right)=$ $\left[-a_{i j}\right]_{i}^{!}\left(i^{-a_{i j}}, j\right)$ is the character of the irreducible $L\left(i^{-a_{i j}}, j\right)$. Indeed, notice that $I_{j}$ only admits flags of type $\left(i^{-a_{i j}}, j\right)$ and the coefficient of $\left(i^{-a_{i j}}, j\right)$ in $\Omega\left(\left[I_{j}\right]^{*}\right)$ is $v^{-\left(\begin{array}{c}-a_{i j} \\ 2\end{array}\right)}\left(-a_{i j}\right)_{i}^{!}=\left[-a_{i j}\right]_{i}^{!}$ where we used that $\left\langle\alpha_{j}, \alpha_{i}\right\rangle=0$.

Similarly, replacing $Q$ by $Q^{\text {op }}$ and $Q_{i j}$ by $Q_{i j}^{\text {op }}$ we see by a similar argument that the unique non-simple projective representation $P_{j}$, the projective cover of the simple $S_{j}$, satisfies $\Omega\left(\left[P_{j}\right]^{*}\right)=$ $\left[-a_{i j}\right]_{i}^{!}\left(j, i^{-a_{i j}}\right)$ is the character of the irreducible $L\left(j, i^{-a_{i j}}\right)$. 
For symmetric types, i.e. equally valued quivers $Q$, Conjecture 8.2 can be seen as a consequence of the geometric construction of Varagnolo-Vasserot and Webster [37, 38].

Theorem 8.4. Conjecture 8.2 holds in the case $d_{i}=d_{j}$ for all $i, j \in Q_{0}$.

Proof. We will begin by fixing some notation roughly following [37]. Let $V$ be a rigid representation of $Q$ and write $\nu=|V|$ for its dimension vector. Let $\mathcal{E}_{\nu}$ be the space of all $Q$ representations on the $\mathbb{N}^{Q_{0}}$ graded vector space underlying $V$. The group $\mathcal{G}_{V}=\prod_{i \in Q_{0}} G L\left(V_{i}\right)$ acts on $\mathcal{E}_{\nu}$ by the standard conjugation action. Write $\mathcal{D}_{G_{V}}\left(\mathcal{E}_{\nu}\right)$ for the $\mathcal{G}_{V}$-equivariant bounded derived category of complexes of sheaves on $\mathcal{E}_{\nu}$. Let $\widetilde{\mathcal{F}}_{\nu}$ denote the variety of all pairs $(x, \phi)$ where $x \in \mathcal{E}_{\nu}$ is a $Q$-representation and $\phi$ is a complete flag of subrepresentations. The group $\mathcal{G}_{V}$ acts on $\widetilde{\mathcal{F}}_{\nu}$ and the projection $\pi: \widetilde{\mathcal{F}}_{\nu} \rightarrow \mathcal{E}_{\nu}$ is a $\mathcal{G}_{V}$-equivariant proper morphism. The variety $\widetilde{\mathcal{F}}_{\nu}$ can be decomposed as a disjoint union of isotypic components $\widetilde{\mathcal{F}}_{\nu}=\bigsqcup_{\mathbf{j} \in \mathbf{W}^{\nu}} \widetilde{\mathcal{F}}_{\mathbf{j}}$ consisting of pairs $(x, \phi)$, where $\phi$ is a flag of type $\mathbf{j}$; this decomposition respects the action of $\mathcal{G}_{V}$. Write $L_{\mathbf{j}} \in \mathcal{D}_{G_{V}}\left(\mathcal{E}_{\nu}\right)$ for the normalized pushforward under the map $\pi$ of the constant sheaf on $\mathcal{F}_{\mathbf{j}}$, i.e. shift the pushforward up by the dimension of $\mathcal{F}_{\mathbf{j}}$.

Theorem 8.5 ([37, Theorem 3.6]). The KLR-algebra $R_{\nu}$ is isomorphic to the Ext-algebra $\operatorname{Ext}_{\mathcal{G}_{V}}^{\bullet^{\prime}}\left(L_{\nu}, L_{\nu}\right)$, where $L_{\nu}=\bigoplus_{\mathbf{j} \in \mathbf{W}^{\nu}} L_{\mathbf{j}}$. Under this isomorphism, the idempotent $e_{\mathbf{j}}$ corresponds to the identity map on $L_{\mathbf{j}}$.

From a $\mathcal{G}_{V}$-variety $\mathcal{Y}$ and a $\mathcal{G}_{V}$-equivariant morphism $\alpha: \mathcal{Y} \rightarrow \mathcal{E}_{\nu}$ we can construct the fiber product $\bigsqcup_{\mathbf{j}} \mathcal{Y} \times \mathcal{E}_{\nu} \mathcal{F}_{\mathbf{j}}$ whose $\mathcal{G}_{V}$-equivariant homology can be identified with $\operatorname{Ext}_{\mathcal{G}_{V}}^{\bullet}\left(L, L_{\nu}\right)$, where $L$ is the pushforward of the constant sheaf on $\mathcal{Y}$ along the morphism $f$ shifted up by the dimension of $\mathcal{Y}$, in particular there is an action of $\operatorname{Ext}_{\mathcal{G}_{V}}\left(L_{\nu}, L_{\nu}\right)$ on the homology. Take $\mathcal{Y}=\mathcal{G}_{V}$ and let $\alpha$ be the action map $\alpha(g)=g \cdot V$ whose image is identified with the dense subset of $\mathcal{E}_{\nu}$ consisting of representations isomorphic to the original rigid representation $V$. In this case the fiber product above is identified with $\bigsqcup_{\mathbf{j}} \mathcal{F}_{\mathbf{j}}(V)$, where the $e_{\mathbf{j}}$ weight space of the $R_{\nu}$ action is exactly the homology of $\mathcal{F}_{\mathbf{j}}(V)$. By Corollary 7.8 and [8, Theorem 6.1.2], the Poincaré polynomial of $\mathcal{F}_{\mathbf{j}}(V)$ is equal to its counting polynomial. Taking into account our choice of shift and that the dimension of $\mathcal{F}_{\mathbf{j}}(V)$ is $\sum_{k<\ell}\left\langle\alpha_{j_{\ell}}, \alpha_{j_{k}}\right\rangle$, it follows that $\Omega\left([V]^{*}\right)$ is the character of the $R_{\nu}$-module $H_{*}^{G_{V}}\left(\bigsqcup_{\mathbf{j}} \mathcal{F}_{\mathbf{j}}(V)\right)$.

It only remains to see that this module is irreducible. First note that $\bigsqcup_{\mathbf{j}} \mathcal{F}_{\mathbf{j}}(V)$ is exactly the fiber of the map $\pi$ over the point $V \in \mathcal{E}_{\nu}$. It follows (as in [6, equation (8.6.24)]) that the JordanHölder multiplicity of a simple $R_{\nu}$-module in any composition series of $H_{*}^{G_{V}}\left(\bigsqcup_{\mathbf{j}} \mathcal{F}_{\mathbf{j}}(V)\right)$ is determined by the stalk over $V$ of the corresponding IC sheaf appearing in $L_{\nu}$. But $\mathcal{G}_{V} \cdot\{V\} \subset \mathcal{E}_{\nu}$ is a locally closed, smooth, irreducible dense subvariety which is $\mathcal{G}_{V}$-equivariantly simply connected. It follows (see [24, Section 1.5]) that there is a unique perverse sheaf on $\mathcal{E}_{\nu}$ whose restriction to $\mathcal{G}_{V} \cdot\{V\}$ is nonzero. In fact, the restriction will be the constant local system on $\mathcal{G}_{V} \cdot\{V\}$ shifted by the dimension of the orbit. Thus there is a unique simple $R_{\nu}$-module appearing in $H_{*}^{G_{V}}\left(\bigsqcup_{\mathbf{j}} \mathcal{F}_{\mathbf{j}}(V)\right)$ and it appears with multiplicity one, as desired.

\subsection{Relation to dual canonical basis conjecture for acyclic quantum cluster algebras}

The corollary below establishes the dual canonical basis conjecture for symmetric types recovering a result of Kimura-Qin [16].

Corollary 8.6. Assume $A$ is a symmetric Cartan matrix and let $w=c^{2}$ for a Coxeter element $c \in W$. Each non-initial cluster monomial of $\mathcal{A}_{v}\left[N^{w}\right]$ is contained in the dual canonical basis. 
Proof. Let $\mathbf{i}_{0}$ be a reduced word for $c$ and consider any valued quiver $(Q, \mathbf{d})$ which admits source adapted sequence $\mathbf{i}_{0}$. Write $\mathbf{i}=\left(\mathbf{i}_{0}, \mathbf{i}_{0}\right)$. From Theorem 5.9 we know that there is a quantum cluster structure on $\mathcal{A}_{v}\left[N^{w}\right]$ and by Theorem 5.2 the elements $X_{V}=\widetilde{\Psi}_{\mathbf{i}}\left([V]^{*}\right) \in$ $\widetilde{\Psi}_{\mathbf{i}}\left(\mathcal{A}_{v}\left[N^{w}\right]\right)$ for $V$ a rigid representation of $(Q, \mathbf{d})$ exactly correspond to non-initial quantum cluster monomials. But we have the factorization $\widetilde{\Psi}_{\mathbf{i}}=\bar{\Psi}_{\mathbf{i}} \circ \Omega$ from Theorem 7.6 which gives $X_{V}=\bar{\Psi}_{\mathbf{i}}\left(\Omega\left([V]^{*}\right)\right)$ is the image of the dual canonical basis element $\Omega\left([V]^{*}\right)$.

In the symmetrizable types it is known that dual canonical basis elements need not have positive multiplicative structure constants, while this is still suspected to hold for skew-symmetrizable quantum cluster algebras. The validity of Conjecture 8.2 for symmetrizable types would suggest the following refinement of the dual canonical basis conjecture for skew-symmetrizable quantum cluster algebras.

Conjecture 8.7. For any Weyl group element $w \in W$ there is a quantum cluster structure on $\mathcal{A}_{v}\left[N^{w}\right]$, where cluster monomials correspond to irreducible characters of KLR representations.

\section{Consequences and further conjectures}

We conclude this note with some ideas of possible continuations or future directions for this approach.

\subsection{Conjectures on irreducible quiver Hecke modules and quantum cluster algebras}

Fix a Weyl group element $w \in W$. It was shown by Lusztig in $[25,26]$ that the dual canonical basis respects the surjection $\mathcal{A}_{v}[N] \rightarrow \mathcal{A}_{v}\left[\overline{N^{w}}\right]$, i.e. there is a unique subset of the dual canonical basis which induces a basis in $\mathcal{A}_{v}\left[\overline{N^{w}}\right]$, we call this the dual canonical basis of $\mathcal{A}_{v}\left[\overline{N^{w}}\right]$. A dual canonical basis element $b$ is called $w$-adapted if $b$ corresponds to an element in the dual canonical basis of $\mathcal{A}_{v}\left[\overline{N^{w}}\right]$.

Lemma 9.1. A dual canonical basis element $b$ is $w$-adapted if and only if $\Psi_{\mathbf{i}}(b) \neq 0$ for a reduced word $\mathbf{i}=\left(i_{1}, \ldots, i_{m}\right)$ of $w$.

Proof. This is an immediate consequence of Theorem 3.2 and the discussion above.

Since in symmetric types irreducible characters for the KLR algebra give dual canonical basis elements, we will say for arbitrary symmetrizable types that an irreducible representation $L$ of $R$ is $w$-adapted if $\bar{\Psi}_{\mathbf{i}}\left(\operatorname{ch}_{v}(L)\right) \neq 0$ for a reduced word $\mathbf{i}=\left(i_{1}, \ldots, i_{m}\right)$ of $w$. Following the cluster algebra philosophy we will call an irreducible character a $w$-adapted coefficient of its image under $\bar{\Psi}_{\mathbf{i}}$ is a single monomial and a $w$-adapted non-coefficient otherwise.

Conjecture 9.2. Each $w$-adapted coefficient is invertible in $\mathcal{A}_{v}\left[N^{w}\right]$ and the set of all $w$-adapted non-coefficients forms a basis of $\mathcal{A}_{v}\left[N^{w}\right]$ over the Laurent ring of coefficients.

From now on we will restrict ourselves to $w$-adapted irreducible representations. To describe an analogue of clusters we make the following observation. Cluster monomials have the property that the product of any two cluster monomials from the same cluster is (up to a power of $v$ ) again a cluster monomial. Thus we make the following translation to the $R$-module language.

Definition 9.3. We define a stably irreducible family of $R$-modules to be a set $\mathbf{L}$ of irreducible modules such that for all $L, L^{\prime} \in \mathbf{L}, L \circ L^{\prime}$ is irreducible and is (up to a grading shift) an element of $\mathbf{L}$. 
Kleshchev-Ram [19] have shown that arbitrary self-inductions of cuspidal irreducible representations are again irreducible, in particular this shows that stably irreducible families exist. Conjecture 8.2 also implies the existence of stably irreducible families, namely the irreducible $R$ modules corresponding to summands of any fixed maximal rigid representation of $(Q, \mathbf{d})$. Since our cluster monomials should be monomials in $m$ variables we make the following definition.

Definition 9.4. For a stably irreducible family $\mathbf{L}$ we call an element $L \in \mathbf{L}$ atomic if there do not exist $L^{\prime}, L^{\prime \prime} \in \mathbf{L}$ such that $L^{\prime} \circ L^{\prime \prime}=L$. Call a stably irreducible family $\mathbf{L} k$-generated if there exists a minimal collection of atomic elements $\left\{L_{1}, \ldots, L_{k}\right\} \subset \mathbf{L}$ so that (up to a grading shift) every element of $\mathbf{L}$ is of the form $L_{j_{1}} \circ \cdots \circ L_{j_{r}}\left(1 \leq j_{\ell} \leq k\right)$ for some $r \geq 1$. In this case we will write $\mathbf{L}=\left\langle L_{1}, \ldots, L_{k}\right\rangle$.

Since the stably irreducible families are naturally ordered by inclusion, we may speak about maximal stably irreducible families of $w$-adapted representations.

Conjecture 9.5. Every maximal stably irreducible family (of w-adapted representations) is mgenerated (where $m$ is the length of $w$ ).

Thus we propose that maximal stably irreducible families should correspond to the clusters of $\mathcal{A}_{v}[N(w)]$, where cluster variables are given by the atomic elements.

Remark 9.6. The restriction to $w$-adapted representations in Conjecture 9.5 seems to be essential. Indeed, in general for infinite-types by allowing arbitrary representations there should exist stably irreducible families generated by any finite number of atomic elements.

Having in mind the analogy with the hypothetical cluster structure on $\mathcal{A}_{v}\left[N^{w}\right]$ we make the following conjectures.

Conjecture 9.7. Suppose each reduced expression for $w$ contains every simple reflection at least once. Then there exist an n-generated stably irreducible collection $\mathbf{C}=\left\langle C_{1}, \ldots, C_{n}\right\rangle$ such that (up to a grading shift) $\mathbf{C} \subset \mathbf{L}$ for every maximal stably irreducible family $\mathbf{L}$ and each $C_{i}$ is atomic in $\mathbf{L}$.

As the notation suggests the $C_{i}$ should be thought of as coefficients in the cluster associated to L. Assume Conjecture 9.7 holds.

Conjecture 9.8. Let $\mathbf{L}=\left\langle L_{1}, \ldots, L_{m-n}, C_{1}, \ldots, C_{n}\right\rangle$ be a maximal stably irreducible family and let $L_{k} \in \mathbf{L}$ be an atomic non-coefficient. Then there exists a unique stably irreducible $R$ module $L_{k}^{\prime} ¥ L_{k}$ such that $\mathbf{L}^{\prime}=\left\langle L_{1}, \ldots, L_{k-1}, L_{k}^{\prime}, L_{k+1}, \ldots, L_{m-n}, C_{1}, \ldots, C_{n}\right\rangle$ is a maximal stably irreducible family different from $\mathbf{L}$.

A proof of Conjecture 9.8 would (essentially, modulo interpreting exchange matrices) complete the monoidal categorification of the cluster structure on $\mathcal{A}_{v}\left[N^{w}\right]$ by KLR algebras.

\section{Acknowledgements}

The author would like to thank Sasha Kleshchev for introducing him to KLR algebras and for leading him in this direction of research. The author would also like to thank Arkady Berenstein for introducing him to Hall algebras and their beautiful properties. Finally, special thanks need to be given to the anonymous referees for helping to solidify the proof of Theorem 8.4. 


\section{References}

[1] Benkart G., Kang S., Oh S., Park E., Construction of irreducible representations over Khovanov-LaudaRouquier algebras of finite classical type, arXiv:1108.1048.

[2] Berenstein A., Group-like elements in quantum groups and Feigin's conjecture, q-alg/9605016.

[3] Berenstein A., Rupel D., Quantum cluster characters of Hall algebras, Selecta Math., to appear, arXiv:1308.2992.

[4] Berenstein A., Zelevinsky A., Quantum cluster algebras, Adv. Math. 195 (2005), 405-455, math.QA/0404446.

[5] Chen X., Xiao J., Exceptional sequences in Hall algebras and quantum groups, Compositio Math. 117 (1999), 161-187.

[6] Chriss N., Ginzburg V., Representation theory and complex geometry, Birkhäuser Boston, Inc., Boston, MA, 1997.

[7] Green J.A., Hall algebras, hereditary algebras and quantum groups, Invent. Math. 120 (1995), 361-377.

[8] Hausel T., Rodriguez-Villegas F., Mixed Hodge polynomials of character varieties, Invent. Math. 174 (2008), 555-624, math.AG/0612668.

[9] Hernandez D., Leclerc B., Quantum Grothendieck rings and derived Hall algebras, J. Reine Angew. Math., to appear, arXiv:1109.0862.

[10] Iohara K., Malikov F., Rings of skew polynomials and Gel'fand-Kirillov conjecture for quantum groups, Comm. Math. Phys. 164 (1994), 217-237, hep-th/9306138.

[11] Jiang Y., Sheng J., Xiao J., The elements in crystal bases corresponding to exceptional modules, Chin. Ann. Math. Ser. B 31 (2010), 1-20, arXiv:0902.1216.

[12] Joseph A., Sur une conjecture de Feigin, C. R. Acad. Sci. Paris Sér. I Math. 320 (1995), 1441-1444.

[13] Kang S., Kashiwara M., Kim M., Symmetric quiver Hecke algebras and $R$-matrices of quantum affine algebras II, arXiv:1308.0651.

[14] Khovanov M., Lauda A.D., A diagrammatic approach to categorification of quantum groups. I, Represent. Theory 13 (2009), 309-347, arXiv:0803.4121.

[15] Khovanov M., Lauda A.D., A diagrammatic approach to categorification of quantum groups. II, Trans. Amer. Math. Soc. 363 (2011), 2685-2700, arXiv:0804.2080.

[16] Kimura Y., Qin F., Graded quiver varieties, quantum cluster algebras and dual canonical basis, Adv. Math. 262 (2014), 261-312, arXiv:1205.2066.

[17] Kleshchev A., Cuspidal systems for affine Khovanov-Lauda-Rouquier algebras, Math. Z. 276 (2014), 691726 , arXiv:1210.6556.

[18] Kleshchev A., Ram A., Homogeneous representations of Khovanov-Lauda algebras, J. Eur. Math. Soc. 12 (2010), 1293-1306, arXiv:0809.0557.

[19] Kleshchev A., Ram A., Representations of Khovanov-Lauda-Rouquier algebras and combinatorics of Lyndon words, Math. Ann. 349 (2011), 943-975, arXiv:0909.1984.

[20] Lampe P., A quantum cluster algebra of Kronecker type and the dual canonical basis, Int. Math. Res. Not. 2011 (2011), 2970-3005, arXiv:1002.2762.

[21] Lampe P., Quantum cluster algebras of type $A$ and the dual canonical basis, Proc. Lond. Math. Soc. 108 (2014), 1-43, arXiv:1101.0580.

[22] Lauda A.D., Vazirani M., Crystals from categorified quantum groups, Adv. Math. 228 (2011), 803-861, arXiv:0909.1810.

[23] Leclerc B., Dual canonical bases, quantum shuffles and q-characters, Math. Z. 246 (2004), 691-732, math.QA/0209133.

[24] Lusztig G., Quivers, perverse sheaves, and quantized enveloping algebras, J. Amer. Math. Soc. 4 (1991), 365-421.

[25] Lusztig G., Introduction to quantum groups, Progress in Mathematics, Vol. 110, Birkhäuser Boston, Inc., Boston, MA, 1993.

[26] Lusztig G., Problems on canonical bases, in Algebraic Groups and their Generalizations: Quantum and Infinite-Dimensional Methods (University Park, PA, 1991), Proc. Sympos. Pure Math., Vol. 56, Amer. Math. Soc., Providence, RI, 1994, 169-176. 
[27] McNamara P.J., Finite dimensional representations of Khovanov-Lauda-Rouquier algebras I: Finite type, arXiv:1207.5860.

[28] Qin F., Quantum cluster variables via Serre polynomials, J. Reine Angew. Math. 668 (2012), 149-190, arXiv:1004.4171.

[29] Ringel C.M., Hall algebras and quantum groups, Invent. Math. 101 (1990), 583-591.

[30] Ringel C.M., Hall algebras revisited, in Quantum Deformations of Algebras and their Representations (Ramat-Gan, 1991/1992; Rehovot, 1991/1992), Israel Math. Conf. Proc., Vol. 7, Bar-Ilan Univ., Ramat Gan, 1993, 171-176.

[31] Rosso M., Quantum groups and quantum shuffles, Invent. Math. 133 (1998), 399-416.

[32] Rouquier R., 2-Kac-Moody algebras, arXiv:0812.5023.

[33] Rouquier R., Quiver Hecke algebras and 2-Lie algebras, Algebra Colloq. 19 (2012), 359-410, arXiv:1112.3619.

[34] Rupel D., On a quantum analog of the Caldero-Chapoton formula, Int. Math. Res. Not. 2011 (2011), $3207-3236$.

[35] Rupel D., Quantum cluster characters of valued quivers, arXiv:1109.6694.

[36] Toën B., Derived Hall algebras, Duke Math. J. 135 (2006), 587-615, math.QA/0501343.

[37] Varagnolo M., Vasserot E., Canonical bases and KLR-algebras, J. Reine Angew. Math. 659 (2011), 67-100, math.RT/0107177.

[38] Webster B., Weighted Khovanov-Lauda-Rouquier algebras, arXiv:1209.2463. 